\title{
Quantitative rescattering theory for laser-induced high-energy plateau photoelectron spectra
}

\author{
Zhangjin Chen, ${ }^{1}$ Anh-Thu Le, ${ }^{1}$ Toru Morishita, ${ }^{2}$ and C. D. Lin ${ }^{1}$ \\ ${ }^{1}$ J. R. Macdonald Laboratory, Physics Department, \\ Kansas State University, Manhattan, Kansas 66506-2604, USA \\ ${ }^{2}$ Department of Applied Physics and Chemistry, University of Electro-Communications, \\ 1-5-1 Chofu-ga-oka, Chofu-shi, Tokyo, 182-8585, Japan and PRESTO, \\ JST Agency, Kawaguchi, Saitama 332-0012, Japan
}

(Dated: April 27, 2022)

\begin{abstract}
A comprehensive quantitative rescattering (QRS) theory for describing the production of highenergy photoelectrons generated by intense laser pulses is presented. According to the QRS, the momentum distributions of these electrons can be expressed as the product of a returning electron wave packet with the elastic differential cross sections (DCS) between free electrons with the target ion. We show that the returning electron wave packets are determined mostly by the lasers only, and can be obtained from the strong field approximation. The validity of the QRS model is carefully examined by checking against accurate results from the solution of the time-dependent Schrödinger equation for atomic targets within the single active electron approximation. We further show that experimental photoelectron spectra for a wide range of laser intensity and wavelength can be explained by the QRS theory, and that the DCS between electrons and target ions can be extracted from experimental photoelectron spectra. By generalizing the QRS theory to molecular targets, we discuss how few-cycle infrared lasers offer a promising tool for dynamic chemical imaging with temporal resolution of a few femtoseconds.
\end{abstract}

PACS numbers: $32.80 . \mathrm{Rm}, 32.80 . \mathrm{Fb}, 42.50 . \mathrm{Hz}$

\section{INTRODUCTION}

Much of our knowledge of the nonlinear interaction of intense laser radiation with atoms and molecules comes from the study of above-threshold ionization (ATI) which is characterized by a sequence of peaks in the electron spectrum, spaced by the photon energy. Since its first observation [1], the subject has been "reinvestigated" many times. In 1987, as sub-picosecond laser pulses became available, it was shown [2] that ATI peaks suffer significant energy shifts and broadening, and each peak breaks up into substructures due to resonance enhancements produced by ponderomotive shifts of states. These substructures are called Freeman resonances. The nature of these Freeman resonances have been carefully investigated, for example for Ar in Wiehle et al. [3]. In recent years, with the introduction of COLTRIM detectors where electrons are measured over almost the whole $4 \pi$ angular region, the two-dimensional (2D) electron momentum spectra or the longitudinal electron momentum spectra of the photoelectrons or the target ions have been reported [4, 5]. These measurements reveal considerable structure not only in the electron energy distributions, but also in the angular distributions. All of these studies focus on low energy electrons which are generated either by multiphoton ionization mechanism or by the tunneling ionization mechanism. According to the "conventional" wisdom, depending on the Keldysh parameter, $\gamma=\sqrt{I_{p} / 2 U_{p}}$ where $I_{p}$ is the ionization energy of the target and $U_{p}$ the ponderomotive energy, if $\gamma$ is larger than one, the ATI electrons are generated by multiphoton pro- cesses, while if $\gamma$ is small, tunneling ionization is responsible for producing the low energy electrons. However, such a distinction is by no means clear-cut. In Ref. [5], the $2 \mathrm{D}$ electron momentum spectra display pronounced fan-like structures even for laser intensities well into the tunneling ionization regime. Theoretical studies of electron momentum spectra [6, 7] obtained from solving the time-dependent Schrödinger equation (TDSE) show that even in the tunneling region, photoelectron spectra show features that can be identified with the absorption of integral number of photons, as revealed by the angular distributions of the electron at fixed energies. These theoretical multiphoton ionization features, when convoluted with the effect of the spatial distribution of intensities in a focused laser beam, can well reproduce the observed experimental 2D electron momentum spectra [8].

While low-energy electrons, with energy less than about $2 U_{p}$, account for the majority of the electrons generated by an intense laser, already since 1993 photoelectrons extending to $10 U_{p}$ or more have also been observed. These electrons, unlike the low energy electrons generated by the nonlinear processes, do not change with the electron energy rapidly, until a new cutoff near about $10 U_{p}$ is reached. They are known as high-energy plateau photoelectrons. Experiments showed that these electrons exhibit pronounced sidelobes not seen in low-energy electrons [9, 10, 11, 12]. These high-energy ATI (HATI) electrons have been interpreted as due to the rescattering process [13]. According to this model, electrons that are freed from the target atom at some well-defined ionization time may be driven back to revisit its parent ion. If 
these returning electrons are backscattered by the target ion, they can be further accelerated by the laser field and emerge as high-energy electrons, reaching up to about $10 U_{p}$. However, the plateau electron spectra, with energies from $4 U_{p}$ to about $10 U_{p}$, are not always similar for different targets. For target like xenon, the plateau is flat, but for other like krypton, the plateau drops steeply as the electron energy increases. These features actually change with peak laser intensities. Further studies of these HATI spectra around 1997 on inert gases discovered that resonantlike enhancements occur in the electron spectra for particular laser intensities. Depending on the inert gas used, separate series of peaks have been observed. These observations have generated a flurry of theoretical interest 14. Models based on analyzing results from solving TDSE [15, 16] and from Floquet theory [17, 18] have been proposed. Others are based on the channel-closing theory [19, 20]. More recent experiments confirmed that these enhancement disappears when laser pulse duration is reduced [10]. Since the HATI yields are four to five orders of magnitude smaller, despite of these experimental investigations, there are few systematic theoretical calculations in the literature. In recent years, HATI electrons have drawn attention again since when they are generated by few-cycle laser pulses, their counts on the left and the right detectors along the laser polarization axis are different. Such asymmetry can be used to determine the absolute value of the carrier-envelopephase (CEP) of the few-cycle pulses [21].

Recently, we investigated the 2D high-energy photoelectron momentum spectra for atomic targets within the single active electron approximation based on the well-known rescattering model [22, 23]. We proposed a quantitative rescattering (QRS) theory [24, 25] where the HATI electrons are modeled as due to the backscattering of the returning electrons by the target ion. According to the QRS, high-energy photoelectron momentum distributions $D(k, \theta)$ is shown to be expressed simply as

$$
D(k, \theta)=W\left(k_{r}\right) \sigma\left(k_{r}, \theta_{r}\right)
$$

where $\sigma\left(k_{r}, \theta_{r}\right)$ is the elastic differential cross sections (DCS) between free electrons, with momentum $k_{r}$, with the target ion. Here $\theta_{r}$ is the scattering angle with respect to the the direction of the returning electrons along the laser polarization axis. In this equation, $W\left(k_{r}\right)$ is interpreted as the momentum distribution of the returning electrons, to be called returning wave packet (RWP) in this paper. The validity of this QRS model has been tested using $D(k, \theta)$ calculated from solving the TDSE, and $\sigma\left(k_{r}, \theta_{r}\right)$ from the standard quantum mechanical scattering theory. Since there is a one-to-one relation between $(k, \theta)$ and $\left(k_{r}, \theta_{r}\right)$, there are a number of important results from the QRS, as reported in our recent papers. It was shown in Morishita et al. 24] and Chen et al. 225] that one can extract elastic scattering cross sections $\sigma\left(k_{r}, \theta_{r}\right)$ between free electrons and atomic ions from the HATI electron momentum spectra. The predictions have been confirmed in three recent experiments [26, 27, 28]. In Chen et al. 29], it was further shown that the momentum distribution $W\left(k_{r}\right)$ of the RWP can be extracted from the second-order strong field approximation (SFA2), and that $W\left(k_{r}\right)$ depends very little on the target (i.e., up to an overall normalization which is related to the total ionization probability). Thus one can use SFA2 to obtain $W\left(k_{r}\right)$. By multiplying it with $\sigma\left(k_{r}, \theta_{r}\right)$, we can use Eq. (1) to obtain accurate high-energy photoelectron momentum distribution $D(k, \theta)$. Since $W\left(k_{r}\right)$ depends mostly on the lasers only, the target dependence of the HATI spectra can thus be explained based on the behavior of the elastic scattering cross sections $\sigma\left(k_{r}, \theta_{r}\right)$. Based on the QRS model, the energy dependence of plateau ATI electrons seen for different targets are easily understood 25]. For a given target but different lasers, the HATI momentum spectra are determined by the RWP. Applying the QRS model to few-cycle pulses, where the RWP varies with the change of the CEP, we have shown that the QRS theory can be easily used to retrieve the absolute value of the CEP [28, 30]. Since nonsequential double ionization of atoms and molecules are understood based on the rescattering mechanism, in Micheau et al. 31] we showed that using the wave packet $W\left(k_{r}\right)$ obtained from the HATI spectra, we can use QRS to obtain nonsequential double ionization yields.

In this paper, we provide the full details of the QRS theory on atomic targets within the single active electron approximation and establish its validity. Clearly our goal is not to limit ourselves to atomic targets only. We would like to study HATI spectra from molecular targets as well, in particular, from transient molecules. Recall that HATI electrons result from backscattering of the returning electrons by the target ion, i.e., electrons that undergo hard collisions with the target. Thus one should be able to retrieve the structure information of the target from the HATI spectra. In fact, we have shown that this is indeed possible for atomic targets already [32]. Since laser pulses of duration of a few femtoseconds are readily available, one can perform pump-probe measurements where the pump pulse initiates a chemical reaction, such that the atomic coordinates of the molecule would evolve in time. Using a probe laser to take HATI spectra at different time delays, one will then have the opportunity to extract the structure of the transient molecule as a function of time from the measured HATI spectra. Thus short laser pulses may serve as a powerful tool for dynamic chemical imaging of transient molecules, with temporal resolution of a few femtoseconds.

The rest of this paper is arranged as follows. In the next section, we discuss how to calculate the HATI electron momentum spectra by solving the TDSE and using the SFA2. We also explain how the elastic differential cross sections are calculated. We then establish the QRS model. The validity of the QRS model is care- 
fully examined in Section III, by testing against results obtained from solving the TDSE. In Section IV we illustrate the application of the QRS model to experimental HATI spectra. To compare with experimental electron energy spectra, we include the laser focus volume effect. We finish the paper with a summary and outlook. We point out that a similar QRS model has been developed for high-order harmonic generation (HHG) [24, 33, 34].

Atomic units are used in this paper unless otherwise noted. We also mention that in all the calculations the CEP is set to zero in this paper.

\section{THEORETICAL BACKGROUND}

The theory part is separated into four sections. Since the concept of rescattering can be understood in classical mechanics, we first consider the classical rescattering theory for an electron in a one-dimensional (1D) monochromatic laser field. Then we discuss the calculations of ATI spectra by solving the TDSE and using the SFA2. For completeness we also include how the elastic scattering cross sections are computed.

\section{A. Classical one-dimensional rescattering theory}

A classical 1D rescattering theory has been discussed by Paulus et al. [13]. Suppose that an electron in the 1D atom is first released at some time $t_{0}$ into a monochromatic laser field $\mathbf{E}(t)=\hat{z} E_{0} \cos \omega t$, the Newton's equation of motion for this system is given by

$$
\ddot{z}(t)=-E_{0} \cos \omega t
$$

consequently the position of the electron at time $t$ reads

$$
\begin{aligned}
z(t)= & z\left(t_{0}\right)+\frac{E_{0}}{\omega^{2}}\left[\cos \omega t-\cos \omega t_{0}\right] \\
& +\left[\frac{E_{0}}{\omega} \sin \omega t_{0}+\dot{z}\left(t_{0}\right)\right]\left(t-t_{0}\right) .
\end{aligned}
$$

If the electron is initially at the origin with zero initial velocity, the time $t_{r}$ at which it returns to the origin satisfies

$$
\cos \omega t_{r}-\cos \omega t_{0}+\omega \sin \omega t_{0}\left(t_{r}-t_{0}\right)=0 .
$$

The electron will never return to the origin if it is ionized before the laser field reaches its peak value while it can return to the origin more than once when it is born after the peak. It has been shown that higher order returns make very small contribution to the yield [29]. We consider only the first return here. The momentum $k_{r}$ of the electron when it first returns to the origin at time $t_{r}$ is

$$
k_{r} \equiv \dot{z}\left(t_{r}\right)=-\frac{E_{0}}{\omega}\left(\sin \omega t_{r}+\sin \omega t_{0}\right) .
$$

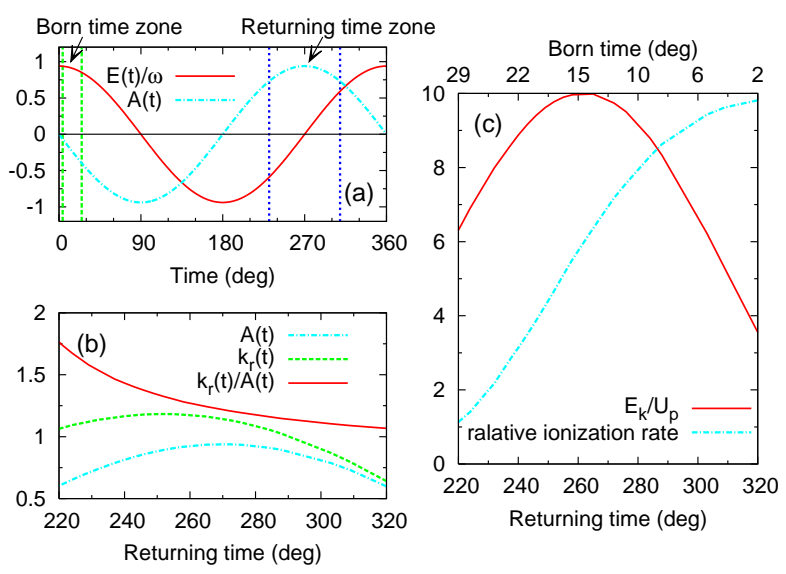

FIG. 1: (Color online) Classical model of a 1D electron in a monochromatic laser field. (a) Electric field and vector potential for a laser at the intensity of $1.0 \times 10^{14} \mathrm{~W} / \mathrm{cm}^{2}$ and wavelength of $800 \mathrm{~nm}$. The born time and returning time zones are marked. (b) The electron velocity $k_{r}$, vector potential $A$, and their ratio at the time of return $\mathrm{t}=\mathrm{t}_{r}$, within the returning time zone. (c) Photoelectron energy after it has been backscattered by an angle $\theta_{r}=180^{\circ}$ against the returning time (bottom horizontal axis). Also shown is the relative ionization rate for electrons released with respect to the born time (top horizontal axis).

In Fig. 1(a) the electric field and the vector potential are plotted vs $\omega t$ for a typical set of laser parameters, say $I_{0}=1.0 \times 10^{14} \mathrm{~W} / \mathrm{cm}^{2}$ and $\lambda=800 \mathrm{~nm}$. The maximum value of the vector potential is $A_{0}=0.94$. In the rescattering model, consider electrons that return within $231^{\circ}<\omega t_{r}<309^{\circ}$. In this region, $A\left(t_{r}\right)>0.78 A_{0}$ and by solving Eq. (3), the corresponding born time is within $4^{\circ}<\omega t_{0}<25^{\circ}$. Both the born time zone and returning time zone are marked in Fig. 1(a). The electron born at time $\omega t_{0}=13^{\circ}$ returns at time $\omega t_{r}=270^{\circ}$. It should be noted that electrons born before $13^{\circ}$ return at the time after $270^{\circ}$ and they follow a long trajectory, while those born after $13^{\circ}$ return before $270^{\circ}$ and they follow a short trajectory.

The vector potential, the velocity of the electron at the return time, and the ratio of the returning velocity to the vector potential are plotted in Fig. 1(b), in the returning time zone. It can be seen that $k_{r}(t) / A(t)$ decrease from 1.76 at $220^{\circ}$ to 1.07 at $320^{\circ}$.

We next assume that the electron undergoes collision with the target ion and elastically scattered by an angle $\theta_{r}$ with respect to its incident direction. For $t \geq t_{r}$, the components of its velocity, along the polarization axis and perpendicular to it, are given by

$$
\begin{aligned}
& \dot{z}(t)=-\frac{E_{0}}{\omega}\left[\sin \omega t-\sin \omega t_{1}+\cos \theta_{r}\left(\sin \omega t_{r} \sin \omega t_{0}\right)\right], \\
& \dot{y}(t)=-\frac{E_{0}}{\omega}\left(\sin \omega t_{r}-\sin \omega t_{0}\right) .
\end{aligned}
$$

From the above equation, the photoelectron energy $E_{k}$ 
measured by the detector outside of the field can be obtained by subtracting the ponderomotive potential, $U_{p}=E_{0}^{2} /\left(4 \omega^{2}\right)$, from the time averaged kinetic energy

$E_{k}=2 U_{p}\left[\sin ^{2} \omega t_{0}+2 \sin \omega t_{r}\left(1-\cos \theta_{r}\right)\left(\sin \omega t_{1}-\sin \omega t_{0}\right)\right] .($

It is easy to find from (77) that, if the electron is born at $\omega t_{0}=14^{\circ}$, it returns to the origin at $\omega t_{r}=265^{\circ}$ when the vector potential almost reaches the maximum, then the photoelectron will have the maximal energy $E_{k}^{\max }=10.007 U_{p}$ provided that the returning electron experiences a backwardscattering of $\theta_{r}=180^{\circ}$. The electron's kinetic energy $E_{k}$ measured by the detector along the polarization axis is shown in in Fig. 1(c), together with the returning time versus with the relative ionization rate of electrons released in the born time zone. The lower horizontal axis in Fig. 1(c) indicates the returning time while the corresponding born time is given on the top. In the QRS model, we investigate backscattered electrons with energies greater than $4 U_{p}$. From Fig. 1(c), it can be seen that electrons return at two different times could have the same kinetic energy except for the time around $265^{\circ}$ at which $E_{k}$ has the maximal value. Actually, electrons which return after $265^{\circ}$ are born before $14^{\circ}$. These electrons have higher ionization rate than those born after $14^{\circ}$. For example, the ionization rate for electrons which are born at $22^{\circ}$ and return around $240^{\circ}$ is about 3 times smaller than electrons born at $2^{\circ}$ and return at $320^{\circ}$.

Let us look back at Fig. 1(b) which shows that the ratio $k_{r}(t) / A(t)$ becomes flatter after a sharp decrease until $240^{\circ}$ and the mean value of $k_{r}(t) / A(t)$ in the returning time range from $240^{\circ}$ to $320^{\circ}$ is about 1.25 . Although the ratio $k_{r}(t) / A(t)$ for returning time less than $240^{\circ}$ deviates more from this mean value, the electron yield from this part is very small, as seen in Fig. 1(c).

\section{B. Method of solving the time-dependent Schrödinger equation}

The method for solving the time-dependent Schrödinger equation has been described in our previous works [6, 8] where we studied the low-energy electron momentum spectra. Much more effort is needed to obtain accurate momentum spectra for high energy electrons. Here we describe the essential steps of the calculations.

We treat the target atom in the single active electron model. The Hamiltonian for such an atom in the presence of a linearly polarized laser can be written as

$$
H=H_{0}+H_{i}(t)=-\frac{1}{2} \nabla^{2}+V(r)+H_{i}(t) .
$$

The atomic model potential $V(r)$ is parameterized in the form

$$
V(r)=-\frac{1+a_{1} e^{-a_{2} r}+a_{3} r e^{-a_{4} r}+a_{5} e^{-a_{6} r}}{r} .
$$

The parameters in Eq. (9) are obtained by fitting the calculated binding energies from this potential to the experimental binding energies of the ground state and the first few excited states of the target atom. The parameters for the targets used in this paper can be found in 35]. For $\mathrm{Kr}$ and $\mathrm{Xe}$, we use the potential given by Garvey et al. [36]. The model potential for a neutral atom can also be expressed as

$$
V(r)=V_{\mathrm{s}}(r)-1 / r,
$$

where $V_{\mathrm{s}}(r)$ is a short-range potential. The atom-field interaction $H_{i}(t)$, in length gauge, is given by

$$
H_{i}=\mathbf{r} \cdot \mathbf{E}(t) .
$$

For a linearly polarized laser pulse (along the $z$ axis) with carrier frequency $\omega$ and the CEP, $\varphi$, the field is taken to have the form

$$
\mathbf{E}(t)=\hat{z} E_{0} \cos ^{2}\left(\frac{\pi t}{\tau}\right) \cos (\omega t+\varphi)
$$

for the time interval $(-\tau / 2, \tau / 2)$ and zero elsewhere. The pulse duration, defined as the full width at half maximum (FWHM) of the intensity, is given by $\Gamma=\tau / 2.75$.

The time evolution of the electronic wavefunction $\Psi(\mathbf{r}, t)$, which satisfies the TDSE,

$$
i \frac{\partial}{\partial t} \Psi(\mathbf{r}, t)=H \Psi(\mathbf{r}, t)
$$

is solved by expanding in terms of eigenfunctions, $R_{n l}(r) Y_{l m}(\hat{\mathbf{r}})$, of $H_{0}$, within the box of $r \in\left[0, r_{\max }\right]$

$$
\Psi(\mathbf{r}, t)=\sum_{n l} c_{n l}(t) R_{n l}(r) Y_{l m}(\hat{\mathbf{r}})
$$

where the radial functions $R_{n l}(r)$ are expanded by the DVR (discrete variable representation) [37, 38, 39] basis set associated with Legendre polynomials, while the $c_{n l}$ are calculated using the split-operator method [40]

$$
\begin{aligned}
c_{n l}(t+\Delta t) \simeq & \sum_{n^{\prime} l^{\prime}}\left\{e^{-i H_{0} \Delta t / 2} e^{-i H_{i}(t+\Delta t / 2) \Delta t}\right. \\
& \left.\times e^{-i H_{0} \Delta t / 2}\right\}_{n l, n^{\prime} l^{\prime}} c_{n^{\prime} l^{\prime}}(t)
\end{aligned}
$$

where the matrix elements are evaluated efficiently by using the DVR quadrature. For short pulses, say, $\Gamma=8 \mathrm{fs}$, and for electron energy as high as $12 U_{p}$, converged results can be obtained by setting $r_{\max }=1200$. Note that in (14), only $m=0$ is taken into account since for linearly polarized laser pulses, contribution to the ionization probability from $m= \pm 1$ is relatively much smaller in comparison to the $m=0$ component.

The photoelectron yield is computed at the end of the laser pulse by projecting the total final wave function onto eigenstates of a continuum electron with momentum $\mathbf{k}$,

$$
D(k, \theta) \equiv \frac{\partial^{3} P}{\partial^{3} \mathbf{k}}=\left|\left\langle\Phi_{\mathbf{k}}^{-} \mid \Psi(t=\tau / 2)\right\rangle\right|^{2}
$$


where the continuum state $\Phi_{\mathbf{k}}^{-}$satisfies the following equation

$$
\left[-\frac{1}{2} \nabla^{2}+V(r)\right] \Phi_{\mathbf{k}}^{-}=\frac{k^{2}}{2} \Phi_{\mathbf{k}}^{-}
$$

\section{Strong field approximation for calculating ATI electron spectra}

While direct solution of the Schrödinger equation in a time-dependent laser field has been widely used, the simpler strong field approximation is of interest for analyzing features of intense laser-atom interactions.

By treating electron-laser interaction as the strong field and electron-target ion interaction as a perturbation, the amplitude for generating a photoelectron with momentum $\mathbf{k}$ is given by

$$
f(\mathbf{k})=f_{1}(\mathbf{k})+f_{2}(\mathbf{k})
$$

where we included the first two terms of the perturbation series only.

In Eq. (18), the first order term $f_{1}(\mathbf{k})$, which is traditional called the strong field approximation (SFA), will be called SFA1, to be distinguished from the second term $f_{2}(\mathbf{k})$, which will be called SFA2. The SFA1 is given by

$$
f_{1}(\mathbf{k})=-i \int_{-\infty}^{\infty} d t\left\langle\chi_{\mathbf{k}}(t)\left|H_{i}(t)\right| \Psi_{0}(t)\right\rangle
$$

where $\Psi_{0}$ is the ground state wavefunction. The Volkov state $\chi_{\mathbf{k}}$ is given by

$$
\left\langle\mathbf{r} \mid \chi_{\mathbf{k}}(t)\right\rangle=\frac{1}{(2 \pi)^{3 / 2}} e^{i[\mathbf{k}+\mathbf{A}(t)] \cdot \mathbf{r}} e^{-i S(\mathbf{k}, t)}
$$

where the action $\mathrm{S}$ is

$$
S(\mathbf{k}, t)=\frac{1}{2} \int_{-\infty}^{t} d t^{\prime}\left[\mathbf{k}+\mathbf{A}\left(t^{\prime}\right)\right]^{2} .
$$

The SFA2 term in (18) is expressed as

$$
\begin{aligned}
f_{2}(\mathbf{k})= & -\int_{-\infty}^{\infty} d t \int_{t}^{\infty} d t^{\prime} \int d \mathbf{p}\left\langle\chi_{\mathbf{k}}\left(t^{\prime}\right)|V| \chi_{\mathbf{p}}\left(t^{\prime}\right)\right\rangle \\
& \times\left\langle\chi_{\mathbf{p}}(t)\left|H_{i}(t)\right| \Psi_{0}(t)\right\rangle .
\end{aligned}
$$

It consists of three time-ordered steps by the electron: tunnel ionization, propagation in the laser field, and elastic scattering with the parent ion. Note that the SFA2 used here is identical to the so-called improved strong field approximation [41, 42].

To evaluate the SFA1 amplitude, we rewrite Eq. (19) as

$$
\begin{aligned}
f_{1}(\mathbf{k}) & =-i \frac{1}{(2 \pi)^{3 / 2}} \int_{-\infty}^{\infty} d t E(t) e^{i S(\mathbf{k}, t)} e^{i I_{p} t} \\
& \times \int d \mathbf{r} e^{-i[\mathbf{k}+\mathbf{A}(t)] \cdot \mathbf{r}} r \cos \theta \Psi_{0}(\mathbf{r})
\end{aligned}
$$

where $I_{p}$ is the ionization potential of the ground state $\Psi_{0}(\mathbf{r})$, and $\theta$ is the polar angle. The ground state wavefunction is calculated from the model potential $V(r)$. To perform integration over space coordinates in (23), we use the identity

$$
e^{-i \mathbf{q} \cdot \mathbf{r}}=4 \pi \sum_{l m} i^{-l} j_{l}(q r) Y_{l m}(\hat{\mathbf{r}}) Y_{l m}^{*}(\hat{\mathbf{q}})
$$

where $j_{l}(q r)$ is the spherical Bessel function and $\cos \theta=$ $\sqrt{4 \pi / 3} Y_{10}(\hat{\mathbf{r}})$. Consequently, the integral over space coordinates can be expressed

$$
\begin{aligned}
\Psi_{0}(\mathbf{q}) & \equiv \int d \mathbf{r} e^{-i \mathbf{q} \cdot \mathbf{r}} r \cos \theta \Psi_{0}(\mathbf{r}) \\
& =4 \pi \sqrt{\frac{4 \pi}{3}} \sum_{l m} i^{-l} Y_{l m}(\hat{\mathbf{q}}) \int d r r^{3} R_{n_{0} l_{0}}(r) j_{l}(q r) \\
& \times \int d \hat{\mathbf{r}} Y_{l m}^{*}(\hat{\mathbf{r}}) Y_{10}(\hat{\mathbf{r}}) Y_{l_{0} m_{0}}(\hat{\mathbf{r}})
\end{aligned}
$$

where the initial state wave function $\Psi_{0}(\mathbf{r})=$ $R_{n_{0} l_{0}}(r) Y_{l_{0} m_{0}}(\hat{\mathbf{r}})$. Due to reason mentioned before, for linearly polarized laser field, we consider $m_{0}=0$ only, and

$$
\begin{aligned}
& \int d \hat{\mathbf{r}} Y_{l m}^{*}(\hat{\mathbf{r}}) Y_{10}(\hat{\mathbf{r}}) Y_{l_{0} m_{0}}(\hat{\mathbf{r}}) \\
& =\sqrt{\frac{3\left(2 l_{0}+1\right)}{4 \pi(2 l+1)}} C\left(1 l_{0} l ; 000\right) C\left(1 l_{0} l ; 000\right) \delta_{m 0}
\end{aligned}
$$

where the C's are the Clebsch-Gordan coefficients. The remaining integration over $r$ is done analytically if hydrogenic wavefunction is used, otherwise it is evaluated numerically. The integration over time is carried out numerically.

For the SFA2 amplitude, we used saddle point approximation for the integral over the momentum $\mathbf{p}$ of the intermediate states and Eq. (22) becomes

$$
\begin{aligned}
f_{2}(\mathbf{k}) & =-\int_{-\infty}^{\infty} d t \int_{-\infty}^{t} d t^{\prime}\left[\frac{2 \pi}{\epsilon+i\left(t-t^{\prime}\right)}\right]^{3 / 2} E\left(t^{\prime}\right) e^{i I_{p} t^{\prime}} \\
& \times e^{-i\left[S\left(\mathbf{p}_{s}, t\right)-S(\mathbf{k}, t)\right]} e^{i S\left(\mathbf{p}_{s}, t^{\prime}\right)} \\
& \times \frac{1}{(2 \pi)^{3}} \int d \mathbf{r}^{\prime} e^{i\left(\mathbf{p}_{s}-\mathbf{k}\right) \cdot \mathbf{r}^{\prime}} V\left(\mathbf{r}^{\prime}\right) \\
& \times \frac{1}{(2 \pi)^{3 / 2}} \int d \mathbf{r} e^{-i\left[\mathbf{p}_{s}+\mathbf{A}\left(t^{\prime}\right)\right] \cdot \mathbf{r}} r \cos \theta \Psi_{0}(\mathbf{r}) . \quad(27)
\end{aligned}
$$

The saddle point is calculated with respect to quasiclassical actions only

$$
\mathbf{p}_{s}\left(t, t^{\prime}\right)=-\frac{1}{t-t^{\prime}} \int_{t^{\prime}}^{t} d t^{\prime \prime} \mathbf{A}\left(t^{\prime \prime}\right)
$$

and the related actions are given by

$$
S\left(\mathbf{p}_{s}, t\right)=\frac{1}{2} \int_{-\infty}^{t} d t^{\prime \prime}\left[\mathbf{p}_{s}\left(t, t^{\prime}\right)+\mathbf{A}\left(t^{\prime \prime}\right)\right]^{2}
$$


and

$$
S\left(\mathbf{p}_{s}, t^{\prime}\right)=\frac{1}{2} \int_{-\infty}^{t^{\prime}} d t^{\prime \prime}\left[\mathbf{p}_{s}\left(t, t^{\prime}\right)+\mathbf{A}\left(t^{\prime \prime}\right)\right]^{2} .
$$

The arbitrary small parameter $\epsilon$ in (27) is introduced to remove possible singularity when $t \rightarrow t^{\prime}$. Actually, the integral (27) converges for the case of initial state $\Psi_{0}(\mathbf{r})$ having $S$ symmetry (as for $\mathrm{H}$ ) while it is divergent for the case of $P$ symmetry (as for Ar) without $\epsilon$ [43]. The Fourier transform of the potential $V(r)$ in (9) is given by

$$
\begin{aligned}
& V(\mathbf{q}) \equiv \int d \mathbf{r} \exp (i \mathbf{q} \cdot \mathbf{r}) V(r) \\
& =-4 \pi\left[\frac{1}{q^{2}}+\frac{a_{1}}{a_{2}^{2}+q^{2}}+\frac{2 a_{3} a_{4}}{\left(a_{4}^{2}+q^{2}\right)^{2}}+\frac{a_{5}}{a_{6}^{2}+q^{2}}\right]
\end{aligned}
$$

It is obvious from (31) that the Fourier transform of $V(r)$ diverges when $q \rightarrow 0$. Therefore, in actual numerical calculations, we multiply the potential by a damping factor

$$
\tilde{V}(r)=V(r) e^{-\alpha r}
$$

to avoid the singularity of the integral. We checked that the results mainly affect the magnitude but not the shape of the HATI spectra.

\section{Elastic differential elastic cross sections}

In this section, we briefly summarize the standard potential scattering theory which has been well documented in the textbooks, see Ref. [44, 45], for example. Without loss of generality, here we address elastic scattering of an electron by a spherical potential $V(r)$ by solving the time-independent Schrödinger equation

$$
\left[\nabla^{2}+k^{2}-U(r)\right] \psi(\mathbf{r})=0
$$

where $U(r)=2 V(r)$ is the reduced potential and $k$ is the electron momentum, related to the incident electron energy by $k=\sqrt{2 E}$. For short-range potential which tends to zero faster then $r^{-2}$ as $r \rightarrow \infty$, the scattering wave function satisfies the asymptotic outgoing wave boundary condition

$$
\psi^{+}(\mathbf{r})_{r \rightarrow \infty}=\frac{1}{(2 \pi)^{3 / 2}}\left[\exp (i k z)+f(\theta) \frac{\exp (i k r)}{r}\right]
$$

where $\theta$ is the polar angle measured from the incident direction. We choose the $z$-axis along the direction of the incident wave vector $\mathbf{k}$.

We solve (33) by expanding the scattering wave function into partial waves,

$$
\psi^{+}(\mathbf{r})=\sqrt{\frac{2}{\pi}} \frac{1}{k r} \sum_{l m} i^{l} e^{i \delta_{l}} u_{l}(k r) Y_{l m}(\hat{\mathbf{r}}) Y_{l m}^{*}(\hat{\mathbf{k}})
$$

where $Y_{l m}$ is a spherical harmonic. The continuum waves are normalized to $\delta\left(\mathbf{k}-\mathbf{k}^{\prime}\right)$. The radial function $u_{l}(k r)$ satisfies

$$
\left[\frac{d^{2}}{d r^{2}}+k^{2}-\frac{l(l+1)}{r^{2}}-U(r)\right] u_{l}(k r)=0 .
$$

For a plane wave, when $U(r)=0$, the radial component $u_{l}(k r) / k r$ in (35) is the standard spherical Bessel function $j_{l}(k r)$.

The radial part of the scattering wave, $u_{l}(k, r)$, has the asymptotic form

$$
u_{l}(k r) \rightarrow \sin \left(k r-\frac{1}{2} l \pi+\delta_{l}\right)
$$

where the phase shift $\delta_{l}$ reflects the influence of the interaction.

The above equations are valid for short-range potentials only. For an electron in a Coulomb potential $V_{\mathrm{c}}=-Z / r$, its full wavefunction can be expanded as

$$
\psi_{\mathrm{c}}^{+}(\mathbf{r})=\sqrt{\frac{2}{\pi}} \frac{1}{k r} \sum_{l m} i^{l} e^{i \sigma_{l}} u_{l}^{\mathrm{c}}(k r) Y_{l m}(\hat{\mathbf{r}}) Y_{l m}^{*}(\hat{\mathbf{k}})
$$

where

$$
\sigma_{l}=\arg [\Gamma(l+1+i \eta)]
$$

is called the Coulomb phase shift with $\eta=-Z / k$. The radial wavefunction $u_{l}^{\mathrm{c}}(k r)$ is solved from

$$
\left[\frac{d^{2}}{d r^{2}}+k^{2}-\frac{l(l+1)}{r^{2}}-\frac{2 \eta k}{r}\right] u_{l}^{\mathrm{c}}(k r)=0 .
$$

However, the expansion (38) does not converge well for a long-range Coulomb potential. For pure Coulomb scattering, the treatment in parabolic coordinates is simpler and the scattering amplitude is given by

$$
f_{\mathrm{c}}(\theta)=-\eta \exp \left(2 i \sigma_{0}\right) \frac{\exp \left\{-i \eta \ln \left[\sin ^{2}(\theta / 2)\right]\right\}}{2 k \sin ^{2}(\theta / 2)} .
$$

For electron-atomic target ion scattering within the single active electron model, the model potential is written as the sum of the Coulomb potential with $Z=1$ and a short-range potential $V_{\mathrm{s}}(r)$, see Eq. (10). For such a modified Coulomb potential problem, the scattering amplitude is given by

$$
f(\theta)=f_{\mathrm{c}}(\theta)+\hat{f}(\theta)
$$

where the first term is the scattering amplitude by the Coulomb potential alone [Eq. (41)], and the second term is given by

$$
\hat{f}(\theta)=\sum_{l=0}^{\infty} \frac{2 l+1}{k} \exp \left(2 i \sigma_{l}\right) \exp \left(i \delta_{l}\right) \sin \delta_{l} P_{l}(\cos \theta)
$$


where the $P_{l}(\cos \theta)$ are Legendre polynomials, and $\delta_{l}$ is the phase shift from the short-range potential. Due to the short range nature, the summation in Eq. (43) can be truncated after some number of partial waves, depending the electron energy. The elastic scattering DCS is then given by

$$
\sigma(k, \theta) \equiv \frac{d P}{d \Omega}=\left|f_{\mathrm{c}}(\theta)+\hat{f}(\theta)\right|^{2} .
$$

For high-energy collisions, one may calculate the differential cross sections using the first Born approximation, or the plane wave Born approximation (PWBA), in which, the DCS is given by

$$
\sigma_{\mathrm{PWBA}}(k, \theta)=\frac{1}{4 \pi^{2}}|V(\mathbf{q})|^{2}
$$

where $\mathbf{q}$ is the momentum transfer and its magnitude is $q=2 k \sin (\theta / 2)$. In PWBA the continuum electron wavefunctions are represented by plane waves. For electrontarget ion collisions, PWBA is not valid even at large collision energies since it neglects the effect of long-range Coulomb interaction as well as the strong short-range potential due to the atomic ion or molecular ion core. In SFA2, scattering of the returning electron wave packet by the target ion is treated by the plane wave Born approximation. This limits the accuracy of using SFA2 in describing the HATI spectra.

\section{QUANTITATIVE RESCATTERING THEORY AND ITS REGION OF VALIDITY}

\section{A. Features of ATI electron energy and momentum spectra}

In the ATI photoelectron energy spectra, it has been well recognized that, after a sharp decrease to around $3 U_{p}$, a plateau exists from 4 to $10 U_{p}$. This universal phenomenon has been observed in experiment, and in the TDSE and SFA calculations as well, see Fig. 2. These figures also show that above about $4 U_{p}$, SFA2 dominates the total electron spectra. Since SFA2 contains a first-order interaction between the electron and the target ion [see Eq. (22)], it is appropriate to attribute that ATI electrons above $4 U_{p}$ are produced by the rescattering processes. In Fig. 2 we note that the HATI spectra for Xe target obtained from TDSE are quite different from $\mathrm{H}$ when they are exposed to the same laser pulse. The HATI plateau in $\mathrm{Xe}$ is very flat. It remains almost constant between 4.5-10 $U_{p}$, while for $\mathrm{H}$ target, in the same energy region the yield drops by a large factor.

Since ATI electrons are produced mostly along the direction of laser polarization (we consider linear polarization only), at photoelectron energies where rescattering becomes dominant depend on the angle of the photoelectrons. In Fig. 3, we show the electron energy distributions for electrons emitted at 0,30,60 and 90 degrees
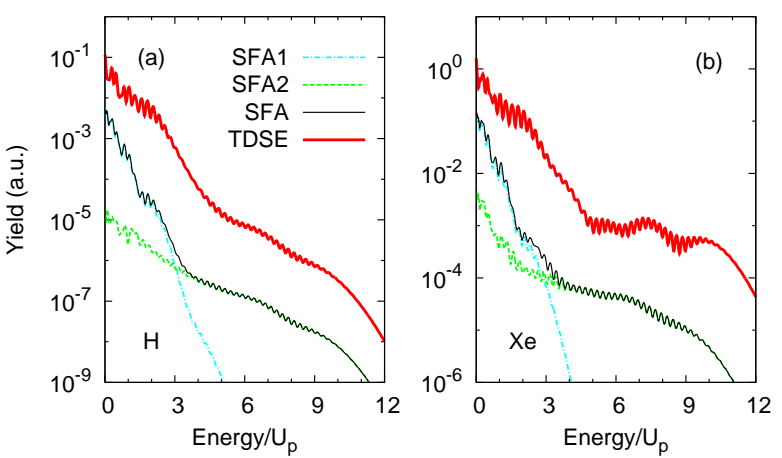

FIG. 2: (Color online) Angle-integrated energy spectra (in units of the ponderomotive energy $U_{p}$ ) calculated from SFA (SFA1 and SFA2) compared with those by solving the TDSE for single ionization of (a) $\mathrm{H}$ and (b) Xe in a 5 fs laser pulse at the intensity of $1.0 \times 10^{14} \mathrm{~W} / \mathrm{cm}^{2}$ with the wavelength of $800 \mathrm{~nm}$.
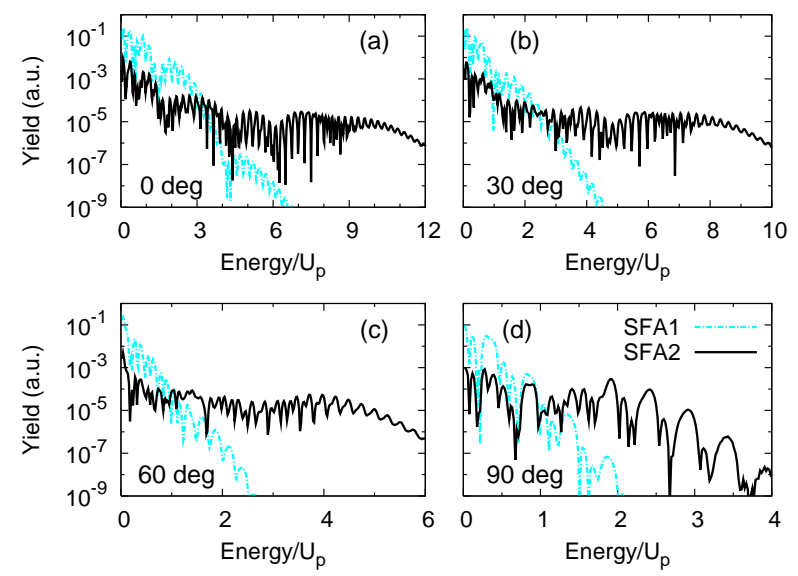

FIG. 3: (Color online) SFA1 and SFA2 energy spectra for single ionization of $\mathrm{Xe}$ in a $10 \mathrm{fs}$ laser pulse at the intensity of $1.0 \times 10^{14} \mathrm{~W} / \mathrm{cm}^{2}$ with the wavelength of $800 \mathrm{~nm}$ at angles of (a) $0^{\circ}$, (b) $30^{\circ}$, (c) $60^{\circ}$, and (d) $90^{\circ}$. The SFA2 dominant region moves to lower energies as the angle is increased.

with respect to laser polarization, calculated using SFA1 and SFA2, respectively. We note that the cutoff for SFA1 where SFA2 becomes dominant shifts from about $4 U_{p}$ at zero degree to $3 U_{p}, 2 U_{p}, 1.5 U_{p}$, respectively, at the angles of 30, 60 and 90 degrees. The laser parameters used in the calculations for Figs. 2 and 3 are given in the captions.

Another method of presenting the angular dependence of electron energy distribution is to display the $2 \mathrm{D}$ momentum distributions. In Fig. 4, the 2D electron momentum distributions obtained from TDSE and SFA2 are shown using the same laser parameters of Fig. 2. Only the large momentum portion is considered since the inside is dominated by SFA1, i.e., the direct ionization. 


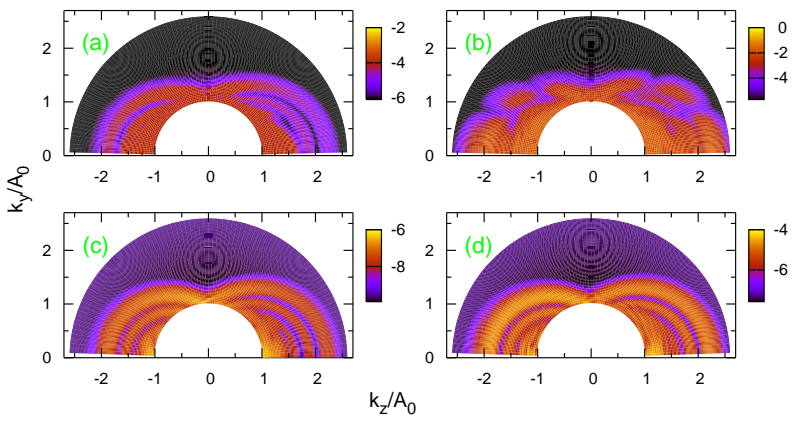

FIG. 4: (Color online) 2D electron momentum distributions for single ionization of $\mathrm{H}$ and $\mathrm{Xe}$ in a $5 \mathrm{fs}$ laser pulse at the intensity of $1.0 \times 10^{14} \mathrm{~W} / \mathrm{cm}^{2}$ with the wavelength of $800 \mathrm{~nm}$. (a) TDSE results for $\mathrm{H}$; (b) TDSE results for Xe; (c) SFA2 results for $\mathrm{H}$; (d) SFA2 results for Xe.

First we note that the appearance of circular "bands" at large momenta for the $\mathrm{H}$ target, in both calculations. The centers of these semi-circular rings are not at the origin, but are shifted along the polarization axis, one on each side. The rings are very similar for $\mathrm{H}$ and $\mathrm{Xe}$ in SFA2, but in TDSE, the intensity distributions show clear structure in Xe, in particular, clear minima at some angles.

\section{B. Extracting electron-target ion elastic differential cross sections from HATI spectra}

High-energy ATI electrons have been observed since 1993 [9, 10, 11, 12, 13]. They were interpreted as due to the backscattering of the returning electrons by the target ion. Indeed classical simulation [13] shows that electrons which return with maximum kinetic energy of $3.17 U_{p}$, if backscattered by 180 degrees, would emerge with kinetic energy of about $10 U_{p}$. Let $A_{0}$ be the peak value of the vector potential of the laser pulse, $U_{p}=A_{0}^{2} / 4$. For an electron that returns at $3.17 U_{p}$, it has momentum $k_{r}=1.26 A_{0}$. For a beam of electrons with momentum $k_{r}$, after elastically scattered, the momentum space forms a circle in 2D (or a surface in 3D) of radius $k_{r}$. Since scattering occurs when the laser field is nearly zero and the vector potential almost has the maximum value $A_{0}$, each electron will gain an additional drift momentum $A_{0}$ as it emerges from the laser field. These electrons were called back rescattered ridge (BRR) electrons in Morishita et al. 24]. The BRR electrons lie on a shifted circle in the photoelectron 2D momentum spectrum. Let the direction of laser polarization be along the $z$-axis, and the $y$-axis perpendicular to it. After the backscattered electron emerges from the laser field, the photoelectron has momentum components

$$
\begin{aligned}
& k_{z}=k \cos \theta= \pm A_{0} \mp k_{r} \cos \theta_{r}, \\
& k_{y}=k \sin \theta=k_{r} \sin \theta_{r} .
\end{aligned}
$$

The upper signs in Eq. (46) refer to the right-side $\left(k_{z}>0\right)$ while the lower ones to the left-side $\left(k_{z}<0\right)$. For backscattering, the angle $\theta_{r}$ is greater than $90^{\circ}$. These two equations can be expressed in vector form $\mathbf{k}= \pm A_{0} \hat{z}+\mathbf{k}_{r}$. This vector relation (the outermost half-circle) is shown in Fig. 5 where the momentum is measured in units of $A_{0}$, and the angles are defined as shown. For $k_{z}>0$, the returning electron enters the target from the right. After a large angle scattering, it is deflected by an angle $\theta_{r}$. As the electron exits the laser field, it makes an angle $\theta$ with respect to the polarization axis.

In Morishita et al. 24], it was argued that if the rescattering picture is correct, the intensity of electrons along the BRR should be proportional to the elastic DCS of the target ion by electrons with incident momentum $k_{r}$. In [24], this model was tested based on the HATI electron spectra calculated from solving the TDSE, for $\mathrm{H}$, $\mathrm{Ne}$, Ar, and Xe targets. For the rare gas atoms, each target is represented by a model potential of the form, Eq. (9). The same model potential was used to calculate HATI electron momentum spectra $D(k, \theta)$ and the elastic DCS, $\sigma\left(k_{r}, \theta_{r}\right)$. By comparing the normalized yield of $D(k, \theta)$ along the ridge of $k_{r}=1.26 A_{0}$ with the DCS, $\sigma\left(k_{r}, \theta_{r}\right)$, it was shown that the two indeed agree very well for the targets tested. The tests have been carried out for different laser intensities and mean wavelengths. Since the $D(k, \theta)$ calculated from solving the TDSE are considered "exact", the test establishes the validity of attributing HATI electron momentum spectra to elastic backscattering of the returning electrons with momentum $k_{r}=1.26 A_{0}$.

The theoretical result of 24] has been limited to BRR electrons only where $k_{r}=1.26 A_{0}$, thus it leaves out a large portion of the HATI spectra where $k_{r}<1.26 A_{0}$. For these lower energy electrons, as shown in Section II.A, the electrons may return to the ion core by following a long- or a short-trajectory. The ratio of the returning electron momentum $k_{r}\left(t_{r}\right)$ vs the vector potential $A_{r}=A\left(t_{r}\right)$ at the time of return, $t=t_{r}$, are shown in Fig. 1(b). Note that the ratio does not change significantly in the time window for those returning electrons that can be backscattered to emerge with energies higher than $4 U_{p}$. Thus we set the relation $k_{r}=1.26 A_{r}$ for all HATI electrons, i.e., Eqs. (46) and (47) are generalized to

$$
\begin{aligned}
k_{z}=k \cos \theta & = \pm k_{r} / 1.26 \mp k_{r} \cos \theta_{r}, \\
k_{y}=k \sin \theta & =k_{r} \sin \theta_{r} .
\end{aligned}
$$

Recall that if one neglects the effect of core potential, the returning electron momentum should be determined by 


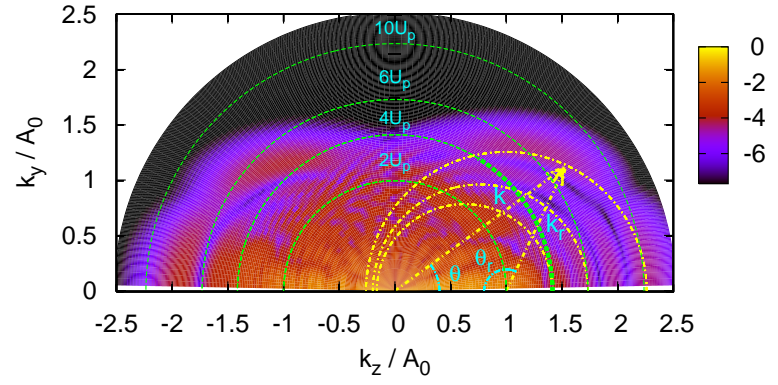

FIG. 5: (Color online) Typical 2D electron momentum distributions (in logarithmic scale): The TDSE calculation is for single ionization of $\mathrm{Ar}$ in a 5 fs laser pulse at the intensity of $1.0 \times 10^{14} \mathrm{~W} / \mathrm{cm}^{2}$ with the wavelength of $800 \mathrm{~nm}$. Photoelectrons of a given energy are represented on a concentric circle centered at the origin. The elastic scattering of a returning electron with momentum $k_{r}$ in the laser field is represented by a partial circle with its center shifted from the origin by $A_{r}=k_{r} / 1.26$. High-energy plateau electrons are obtained via large-angle backscattering only. See text.

the difference of the vector potentials at the return time and the born time. From Fig. 1(a), the range of born time is very narrow, thus the same relation between $k_{r}$ and $A_{r}$ for all HATI electrons is a good approximation. With this model, the center of the circle for each momentum $k_{r}$ is shifted by $k_{r} / 1.26$. This has important implications since the laser parameters such as peak intensity or wavelength do not enter explicitly in Eq. (48) or (49) any more. How good is this model? We test its validity using accurate numerical results from TDSE calculations.

Recall that the validity of Eq. (11), Eqs. (48) and (49), has been fully tested for the case of short pulses and for $k_{r}=1.26 A_{0}$ in [24]. In Fig. 6(a), we show the theoretically calculated DCS for Ar at $k_{r}=1.22$ and compare with the DCS extracted from HATI momentum spectra obtained from solving the TDSE for Ar target. In one case we use a five-cycle pulse with $800 \mathrm{~nm}$ mean wavelength, and peak intensity of $1.0 \times 10^{14} \mathrm{~W} / \mathrm{cm}^{2}$. This is the same as the BRR discussed in [24]. In another case, the peak intensity used is $1.4 \times 10^{14} \mathrm{~W} / \mathrm{cm}^{2}$. The BRR electron momentum for the latter is 1.32 . If we extract the DCS from the HATI spectra of the latter at $k_{r}=1.22$, as seen from Fig. 6(a), the results are still quite good.

Up to now, we have focused on short laser pulses. For longer pulses, the electrons generated from different optical cycles of the laser can interfere. Such interference would result in the well-known ATI peaks which are separated by the photon energy of the laser. From Fig. 5, along $k_{r}=$ constant, the photoelectron energy changes as $\theta_{r}$ is varied. Thus the assumption that $W\left(k_{r}\right)$ is constant along a fixed $k_{r}$ is no longer correct because of the interference in the wave packet. However, the interference effect is well behaved. In Fig. 6(b), we show the

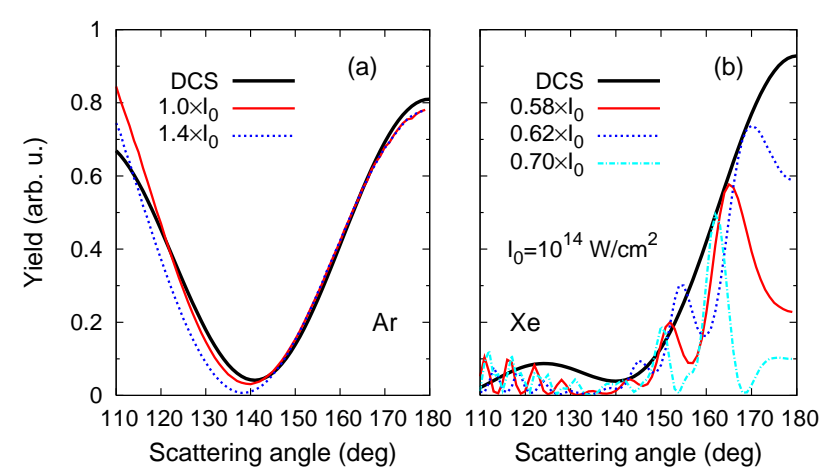

FIG. 6: (Color online) Electron yield extracted for fixed $k_{r}$ from the 2D electron momentum distributions for Ar and Xe by solving the TDSE compared with the corresponding elastic scattering DCS's (thick black solid lines). (a) For the target of $\mathrm{Ar}$ and $k_{r}=1.22$ by a 5 -cycle laser pulse with the wavelength of $800 \mathrm{~nm}$ at intensities of 1.0 and $1.4 \times 10^{14} \mathrm{~W} / \mathrm{cm}^{2}$, respectively; (b) For the target of Xe and $k_{r}=0.92$ by a 8 cycle laser pulse with the wavelength of $800 \mathrm{~nm}$ at intensities of $5.8,6.2$ and $7.0 \times 10^{13} \mathrm{~W} / \mathrm{cm}^{2}$, respectively.

DCS for $\mathrm{e}-\mathrm{Xe}^{+}$at $k_{r}=0.92$. We also extracted the DCS at the same $k_{r}=0.92$ using the HATI spectra generated by three lasers, with peak intensity of 7.0,6.2, and 5.8 in units of $1.0 \times 10^{13} \mathrm{~W} / \mathrm{cm}^{2}$, for $800 \mathrm{~nm}$ lasers of durations of 8 cycles. By assuming a constant wave packet $W\left(k_{r}\right)$, the "extracted" DCS oscillates but the peak positions of the oscillation still follow the DCS quite accurately (after normalized). Such oscillations appear to be worrisome in attempts to extract DCS from HATI spectra using longer laser pulses. However, as will be shown in Section IV.D, this is not a problem for extracting DCS from experimental HATI spectra since experiments "intrinsically" integrate electron spectra generated over a distribution of laser intensities. More discussions on the oscillations of the wave packet are given in the next subsection.

\section{Extracting rescattering wave packet from the HATI spectra}

Given the relation between $(k, \theta)$ and $\left(k_{r}, \theta_{r}\right)$ in Eqs. (48) and (49), in general one can write $D(k, \theta)=$ $W\left(k_{r}, \theta_{r}\right) \sigma\left(k_{r}, \theta_{r}\right)$. If the rescattering concept is meaningful for a given $k_{r}$, we expect $W\left(k_{r}, \theta_{r}\right)=W\left(k_{r}\right)$, i.e., the rescattering wave packet distribution is independent of scattering angles. To illustrate this point, we calculate

$$
W\left(k_{r}, \theta_{r}\right)=D(k, \theta) / \sigma\left(k_{r}, \theta_{r}\right),
$$

where $D(k, \theta)$ is obtained from TDSE and $\sigma\left(k_{r}, \theta_{r}\right)$ from Eq. (44). The results for $W\left(k_{r}, \theta_{r}\right)$ are shown for the angular range of $\theta=155^{\circ}$ to $180^{\circ}$ in Figs. 7(a) and 

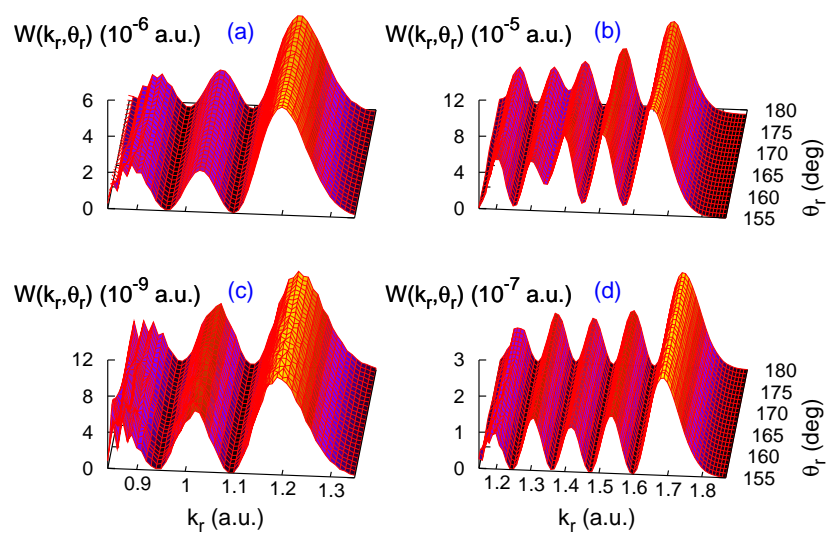

FIG. 7: (Color online) Right-side wave packets $\left(k_{z}>0\right)$ extracted from the TDSE and SFA2 electron momentum distributions for single ionization of Ar in a 5 fs laser pulse with the wavelength of $800 \mathrm{~nm}$. (a,c) TDSE and SFA2 results at the intensity of $1.0 \times 10^{14} \mathrm{~W} / \mathrm{cm}^{2}$. (b,d) TDSE and SFA2 results at the intensity of $2.0 \times 10^{14} \mathrm{~W} / \mathrm{cm}^{2}$.

7(b), for two intensities (with other laser parameters given in the figure), respectively. It is quite clear that there is little angular dependence of $\theta_{r}$ such that we can write $W\left(k_{r}, \theta_{r}\right)=W\left(k_{r}\right)$. We emphasize that this relation is based on computational results where $D(k, \theta)$ and $\sigma\left(k_{r}, \theta_{r}\right)$ are calculated "exactly". The correctness of $W\left(k_{r}, \theta_{r}\right)=W\left(k_{r}\right)$ justifies the relation in Eqs. (48) and (49) and it provides a strong statement of the QRS model for HATI electron momentum spectra, as stated in Eq. (1) in the Introduction. Note that $W\left(k_{r}\right)$ is extracted from $D(k, \theta)$ at the end of the laser pulse, thus $W\left(k_{r}\right)$ includes all the quantum interference due to the long- and short-trajectory electrons, and interference due to wave packets generated from different optical cycles. In Figs. 7(a,b), the pulse duration is 5 fs. The oscillation in the wave packet is due to interference of long- and short-trajectory electrons that return with the same $k_{r}$. As the laser intensity increases, the oscillations become faster. This increase of oscillations can be easily understood based on the SFA2. Note that there are two wave packets, $W\left(k_{r}\right)$, one from the left and the other from the right toward the target.

As discussed earlier, in strong field approximation, SFA2 dominates over SFA1 for HATI electrons with energies above $4 U_{p}$, see Fig. 2. Since rescattering is included in SFA2, we also check whether separation similar to Eq. (11) also applicable to $D(k, \theta)$ calculated from SFA2. In SFA2 the elastic scattering of RWP with the target ion is treated to first order only, thus the corresponding $\sigma\left(k_{r}, \theta_{r}\right)$ is calculated using the plane-wave Born approximation, Eq. (45). Following the same procedure as for the TDSE results, we extract the RWP from SFA2. The results are shown in Figs. 7(c) and 7(d) for the same two sets of lasers used in the TDSE calcula-
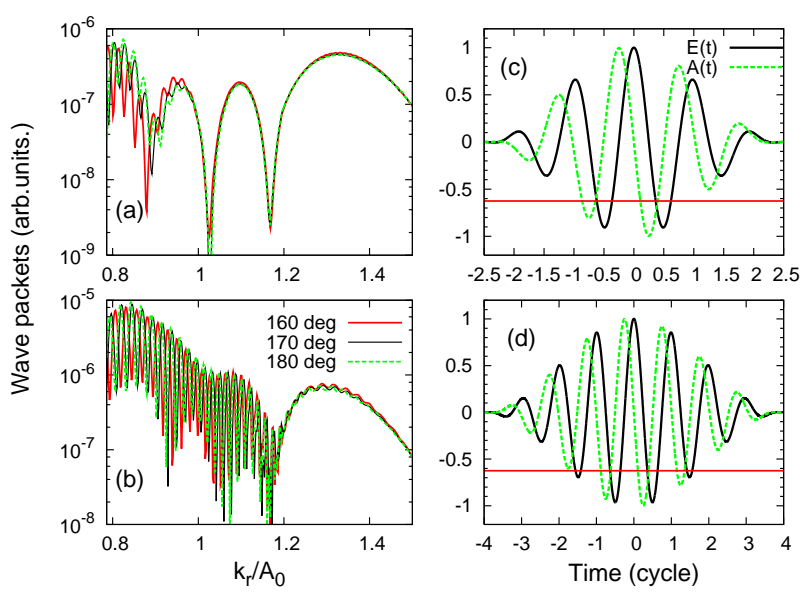

FIG. 8: (Color online) (a) Right-side wave packets $\left(k_{z}>\right.$ 0 ) extracted from TDSE for single ionization of Ar in a 5cycle pulse with the wavelength of $800 \mathrm{~nm}$ at the intensity of $1.0 \times 10^{14} \mathrm{~W} / \mathrm{cm}^{2}$ at $\theta_{r}=160^{\circ}, 170^{\circ}$ and $180^{\circ}$, respectively; (b) Same as (a) but for an 8-cycle pulse; (c,d) Electric field and vector potential used in $(\mathrm{a}, \mathrm{b})$, respectively. The peak values of both $E(t)$ and $A(t)$ are normalized to 1 . The horizontal lines in $(\mathrm{c}, \mathrm{d})$ are for $A_{r}=0.625 A_{0}$.

tions. Comparing Figs. 7(a,c) and Figs. 7(b,d), respectively, we note that the RWP's extracted from SFA2 and from TDSE have very similar shape. After normalization, their dependence on $k_{r}$ is nearly identical for the same laser. The absolute value of $W\left(k_{r}\right)$ from SFA2 is smaller in general since ionization yield calculated using strong field approximation is smaller in general. The similarity of $W\left(k_{r}\right)$ from SFA2 and from TDSE also allows us to interpret the increase of oscillations of RWP as the laser intensity is increased, as seen in Fig. 7. This increase can be traced to the actions $S$, Eqs. (29) and (30), whose values increase quadratically with the vector potential, or linearly with the ponderomotive energy $U_{p}$ and time duration of the pulse.

For longer pulses, the RWP should reflect the interference from different optical cycles. Consider the wave packets shown in Figs. $8(\mathrm{a}, \mathrm{b})$, generated by an $800 \mathrm{~nm}$, peak intensity of $1.0 \times 10^{14} \mathrm{~W} / \mathrm{cm}^{2}$, but one with 5 , and the other 8 optical cycles, respectively. The corresponding $E$-field and $A$-vector are shown to the right. The horizontal lines in Figs. 8(c,d) are for $A_{r}=0.625 A_{0}$. Electrons return with this $A_{r}$ have $k_{r}=1.26 A_{r}$ that can be back rescattered to reach HATI energy of $4 U_{p}$. Note that $A_{r}$ determines the photoelectron energy, but the yield is determined by the electric field at about $3 / 4$ cycles earlier. Thus for the 5-cycle pulse, HATI electrons are generated from one optical cycle only, and the oscillation seen in Fig. 8(a) is due to interference from longand short-trajectory electrons. For the 8-cycle pulse, at least two optical cycles make contributions to the HATI spectra, thus interference seen in Fig. 8(b) becomes much more numerous. The fast oscillations from electrons gen- 
erated at different optical cycles overwhelm the slower oscillations from electrons generated within the same cycle, such that the long- and short-trajectory interference is seen as the oscillation of the envelope in $W\left(k_{r}\right)$. As the pulse duration increases, the momentum distribution of the RWP will become flatter except for the cutoff region. As demonstrated in Fig. 8(b), the finer oscillations in the RWP extracted from different angles $\theta_{r}$ will not be the same. However, the envelope of the wave packet is independent of $\theta_{r}$, such that a single wave packet is still meaningful. More examples can be seen in [29].

\section{Target independence of the shape of the returning electron wave packet}

The fact that the $W\left(k_{r}\right)$ extracted from SFA2 is similar in shape to that extracted from TDSE is again a consequence of the validity of the rescattering model. While the absolute returning electron yield is determined by the initial tunnel ionization rate, its momentum distribution $W\left(k_{r}\right)$ is determined almost entirely by the laser field. In SFA2, this interaction is fully included. The electrontarget ion interaction, which is included in TDSE, affects $W\left(k_{r}\right)$ weakly only since the returning electron spends most of the time away from the target ion where the field is dominated by the laser's electric field.

The fact that $W\left(k_{r}\right)$ can be obtained from SFA2 reasonably accurately has a far-reaching implication. Before discussing such implications, first we look more carefully at how well the $W\left(k_{r}\right)$ obtained from TDSE and SFA2 agree, for different target atoms. In Figs. 9(a-d) we show these RWP's generated by an 800nm, 8-cycle, peak intensity $1.0 \times 10^{14} \mathrm{~W} / \mathrm{cm}^{2}$ laser pulse for $\mathrm{H}$, Ar and Xe targets. We show the "left" and "right" RWP's which are different for the short pulse used. To first order, all the wave packets on the left are similar, and all the wave packets on the right are similar. A more careful examination reveals that there are differences. Among the different targets calculated using TDSE, we note that the distribution of $W\left(k_{r}\right)$ tends to shift to higher $k_{r}$ as the ionization energy increases. This is expected since the returning electron is seeing a more attractive potential from the ion core, and the effect is bigger for lower energy electrons. Still the effect is only a few percents. For longer pulses or higher intensities, see Figs. 9(e,f), the wave packets at lower momenta are mostly very flat where small shift of individual peaks is not very important. Due to the small difference of the RWP on the target for a given laser pulse, the wave packet can be generated using SFA2 from a hydrogenlike target, with the effective charge chosen so that it gives identical binding energy of the target.
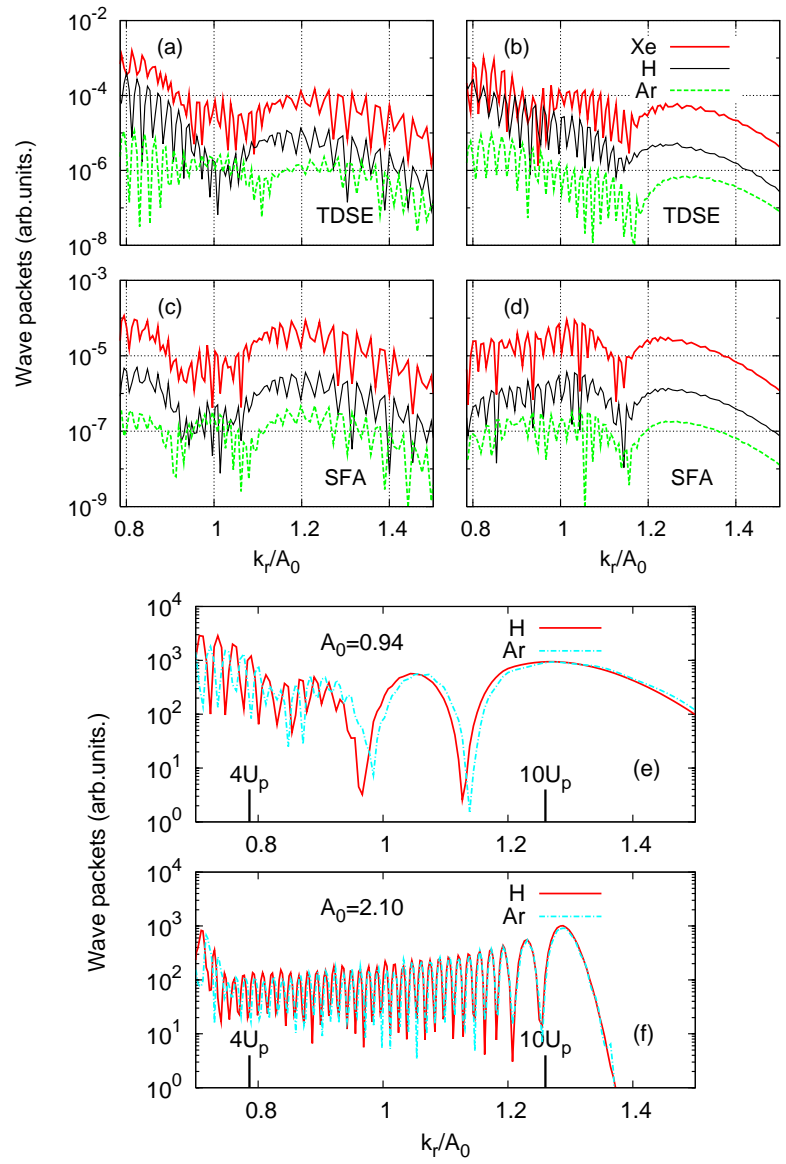

FIG. 9: (Color online) (a) Left-side wave packets $\left(k_{z}<0\right)$ extracted from TDSE for single ionization of $\mathrm{H}, \mathrm{Ar}$ and Xe in a 8-cycle pulse at the peak intensity of $1.0 \times 10^{14} \mathrm{~W} / \mathrm{cm}^{2}$ with wavelength of $800 \mathrm{~nm}$, respectively; (b) Same as (a) but for the right-side $\left(k_{z}>0\right)$; (c) Same as (a) but from SFA2; (d) Same as (b) but from SFA2; (e) Right-side wave packets extracted from SFA2 for single ionization of $\mathrm{H}$ and $\mathrm{Ar}$ in a 5 -cycle laser pulse at the intensity of $1.0 \times 10^{14} \mathrm{~W} / \mathrm{cm}^{2}$ with the wavelength of $800 \mathrm{~nm}$; (f) same as (e) but for intensity of $2.1 \times 10^{14} \mathrm{~W} / \mathrm{cm}^{2}$ and wavelength of $2000 \mathrm{~nm}$.

\section{E. Elastic electron-ion differential cross sections at large angles}

Elastic and inelastic scattering cross sections between free electrons and atomic ions have been studied in crossbeam or merged-beam experiments [4]. In such experiments, a well-collimated electron beam with precisely defined energy is prepared. For neutral atomic or molecular targets which can be placed in a gas cell, there have been lots of experimental and theoretical investigations in the past half a century. These studies tend to focus on sharp features like Feshbach resonances which often require a careful treatment of electron correlation effects. For the HATI spectra, the returning electron is described by a wave packet which has a broad momentum distribution. Here we tend to focus on the broader energy and 

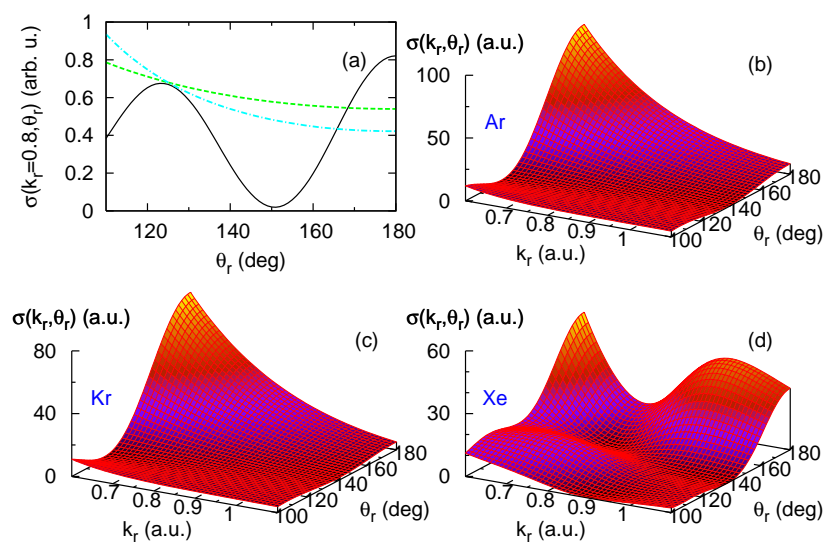

FIG. 10: (Color online) (a) DCS's for Xe at $k_{r}=0.8$ in the angular range of $110^{\circ}-180^{\circ}$. Solid line: "exact" scattering wave result [Eq. (42)]; broken line: PWBA [Eq. (45)]; Chain line: Coulomb wave result [Eq. (41)]. (b,c,d) 2-dimensional "exact" scattering wave results of the DCS's for Ar, Kr and Xe, respectively.

the angular dependence, and neglect the many-electron effects to first order. Within this model, the calculation of elastic scattering cross sections is quite simple, as described in Section II.D.

In Fig. 10(a) we compare the DCS's calculated for Xe at the incident momentum of $k_{r}=0.8$ for scattering angles from $110^{\circ}-180^{\circ}$, where the continuum wave functions are represented by "exact" scattering waves, by plane waves and by Coulomb waves, respectively. The DCS's are normalized near about $130^{\circ}$. We note that the DCS calculated from the PWBA is rather featureless, so is the DCS calculated using Coulomb wavefunctions, each drops monotonically with increasing scattering angle. On the other hand, the DCS calculated from the scattering wave shows complicated pattern which is the well-known Ramsauer-Townsend electron diffraction. This illustrates that both plane wave and Coulomb wave are very poor approximation for describing electron-ion collisions, especially for electrons which undergo large-angle scattering. For such large deflection angles, the electron has to penetrate the ion core, thus seeing the short-range part of the potential. Without scattering waves, the diffraction by the strong potential $V_{\mathrm{s}}(r)$ is neglected and the HATI spectra cannot be correctly reproduced.

In Figs. 10(b-d) we show $\sigma\left(k_{r}, \theta_{r}\right)$ for $\mathrm{Ar}, \mathrm{Kr}$ and Xe for $k_{r}$ from 0.6 to 1.1. The complicated structure, and the increase of cross sections close to $180^{\circ}$, are quite evident for all three targets. Such structure would not appear if $\mathrm{H}$ is used as the target. For the large angles discussed here, the minima in the DCS come from the interference of contributions from several partial waves in the scattering by the short range potential. At large angles, the Coulomb scattering amplitude [See Eq. (41)] is relatively small. The interference of the two amplitudes in Eq. (42) can also produce interference minimum shown in typical textbook examples [44], but such minimum occurs at smaller angles. (See the two minima in the DCS for $\mathrm{e}^{-}+\mathrm{Ar}^{+}$collisions in Fig. 4(c) of [28] where the large-angle minimum at $140^{\circ}$ was derived from the HATI spectra and the small-angle minimum at $89^{\circ}$ was observed from electron- $\mathrm{Ar}^{+}$colliding beam experiment [46].)

The strong dependence of $\sigma\left(k_{r}, \theta_{r}\right)$ on the target potential shows that HATI spectra can be obtained accurately only if electron-ion scattering is treated accurately in the nonlinear interactions of lasers with atoms and molecules. Thus TDSE calculations using approximations where the singularity of the Coulomb core potential is regularized should be handled with care. Such regularized models have less effect on the total ionization yield or the electron spectra at low energies, but it will affect the HATI spectra since these electrons undergo close collisions with the target core. This also implies that strong field calculations where continuum electrons are treated at the level of plane waves (as in SFA2), Coulomb waves (such as using Coulomb Volkov states), or in the eikonal approximation, will not be adequate for the description of the HATI spectra.

\section{F. The practical quantitative rescattering model and its validity}

Based on the established validity of the QRS model using TDSE and SFA2, we now propose a practical QRS model for obtaining HATI electron momentum spectra. We would apply this model to electron energies above about $4 U_{p}$ for the total electron energy spectra. For the energy dependence at each fixed angle, the lower energy limit where this theory applies can be relaxed, as illustrated in Fig. 3.

In the practical QRS model, the HATI momentum distribution $D(k, \theta)$ is calculated using Eq. (1). We obtain the DCS using Eq. (44) and the returning electron wave packet from

$$
W\left(k_{r}\right)=D_{\mathrm{SFA} 2}(k, \theta) / \sigma_{\mathrm{PWBA}}\left(k_{r}, \theta_{r}\right),
$$

where $D_{\mathrm{SFA} 2}(k, \theta)=\left|f_{2}\right|^{2}$ and $f_{2}$ is calculated from Eq. (27), and $\sigma_{\mathrm{PWBA}}\left(k_{r}, \theta_{r}\right)$ is calculated from Eq. (45). The relation between $(k, \theta)$ and $\left(k_{r}, \theta_{r}\right)$ are given by Eqs. (48) and (49). To obtain $W\left(k_{r}\right)$ from Eq. (51), we need to do SFA2 calculation at one angle $\theta_{r}$ only. We typically use $\theta_{r}=170^{\circ}$.

Since $W\left(k_{r}\right)$ is nearly independent of the target for a given laser pulse, we can also perform SFA2 calculation using hydrogenic potential with an effective charge that reproduces the binding energy of the target atom. We emphasize that using the QRS, the absolute yield is not obtained. This is also true for most of the energy and 


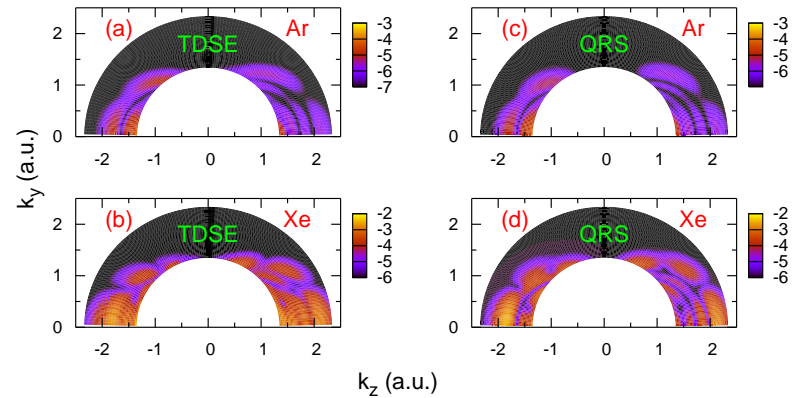

FIG. 11: (Color online) Logarithmic photoelectron 2D momentum distributions by a 5-cycle pulse at the peak intensity of $1.0 \times 10^{14} \mathrm{~W} / \mathrm{cm}^{2}$ with wavelength of $800 \mathrm{~nm}$. (a) TDSE results for Ar; (b) QRS results for Ar; (c) TDSE results for $\mathrm{Xe}$; (d) QRS results for Xe.

momentum spectra reported in experiments. Using the practical QRS model, the calculation of HTAI spectra can be a factor of thousands faster compared to TDSE calculations. However, this is useful only if we can show that the QRS reproduces the HATI momentum and energy spectra at the level comparable to TDSE results.

In Fig. 11 we show the 2D electron momentum spectra calculated using QRS and TDSE for Ar and Xe using the laser parameters indicated in the figure. Recall that for short pulses the left and right wave packets have to be calculated separately. One can see the overall agreement between the QRS and TDSE calculations. In making color plots we renormalize the spectra and the same color schemes are used in the figures. Since the same laser is used in the calculations for both Ar and Xe, according to the QRS, the difference in Figs. 11(c,d) are almost entirely due to the difference in the DCS [see Figs. 10(b,d)] by the returning electrons colliding with the target ions of $\mathrm{Ar}$ and Xe, respectively.

To display the comparison quantitatively, we show the total electron energy spectra obtained from TDSE and those from QRS for energies above $4 U_{p}$. In Fig. 12(a), the electron energy spectra are obtained by integrating the momentum spectra in Fig. 11. The QRS and TDSE results agree quite well above $4 U_{p}$. For Ar, we see some discrepancy close to $4 U_{p}$. In Fig. 12(b), we compare the total electron spectra using lasers of three different wavelengths, with the same number of cycles, but with the intensity adjusted such that $U_{p}=6 \mathrm{eV}$ for each pulse, and the Keldysh parameter is 1.14. The electron spectra calculated from TDSE are placed on the absolute scale, while the QRS results are normalized individually to achieve best agreement. Among the three cases, the DCS's are the same. Even though the returning electron wave packets cover the same range of momentum, the
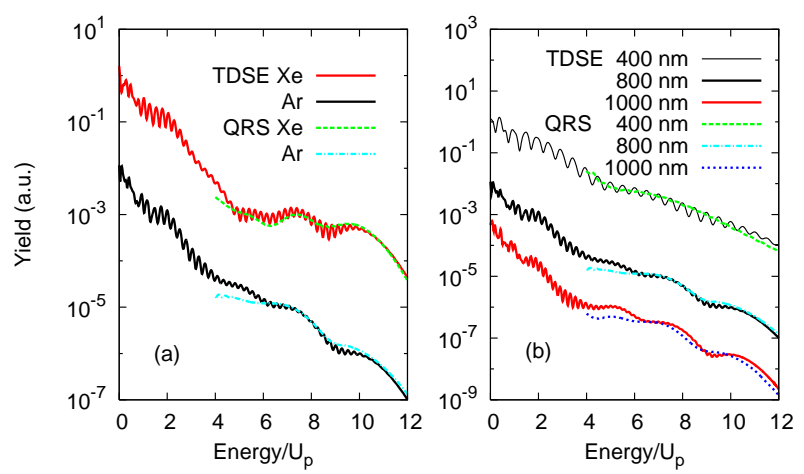

FIG. 12: (Color online) Comparison of angle-integrated photoelectron energy spectra from TDSE and QRS. The TDSE spectra are plotted from 0 to $12 U_{p}$ and the QRS results are shown from 4 to $12 U_{p}$. The QRS spectra are relative and normalized to TDSE results at high energies. (a) Single ionization of $\mathrm{Ar}$ and $\mathrm{Xe}$ in a 5-cycle pulse at the peak intensity of $1.0 \times 10^{14} \mathrm{~W} / \mathrm{cm}^{2}$ with wavelength of $800 \mathrm{~nm}$; (b) Single ionization of $\mathrm{Ar}$ in a 5 -cycle pulse at peak intensities of 4.0, 1.0 and $0.64 \times 10^{14} \mathrm{~W} / \mathrm{cm}^{2}$ with wavelengths of 400,800 and $1000 \mathrm{~nm}$, respectively.

interference features in $W\left(k_{r}\right)$ are different. Such interference features are easily reproduced in the wave packet calculated from SFA2. For $400 \mathrm{~nm}$, the TDSE results show clear ATI peaks which are not reproduced in the QRS model, as explained in Section III.D. For additional examples of the comparison between the QRS model and TDSE, see Chen et al. 25].

\section{COMPARING QRS MODEL WITH EXPERIMENTAL ATI ELECTRON SPECTRA}

\section{A. Volume effect in experimental ATI spectra}

In the previous Section we compared the HATI spectra calculated using TDSE and QRS for a given laser pulse with single intensity. Experimentally, the intensity distribution of a focused laser beam is not uniform in space. The HATI electrons are collected from the whole focused volume. Thus to compare with experimental HATI spectra, theoretical calculations must include the volume effects [3, 8]. For a peak intensity $I_{0}$ at the focal point, the yield of the photoelectrons with momentum $\mathbf{k}$ should be

$$
S\left(\mathbf{k}, I_{0}\right)=\rho \int_{0}^{I_{0}} D_{I}(k, \theta)\left(\frac{\partial V}{\partial I}\right) d I
$$

where $\rho$ is the density of atoms in the chamber, $D_{I}(k, \theta)$ denotes the momentum distribution for a single intensity $I$ and $(\partial V / \partial I) d I$ represents the volume of an isointensity shell between $I$ and $I=I+d I$ defined in [47] 
for a Lorentzian (propagation direction) and a Gaussian (transverse direction) beam profile. We use the trapezoidal rule for the integration over intensity with sufficiently small step size of $0.01 \times 10^{14} \mathrm{~W} / \mathrm{cm}^{2}$.

In the QRS calculations, we obtain the volumeintegrated returning electron wave packet using Eq. (11) since the DCS does not depend on the laser intensity. Consequently, Eq. (52) becomes

$$
S\left(\mathbf{k}, I_{0}\right)=\bar{W}_{I_{0}}\left(k_{r}\right) \sigma\left(k_{r}, \theta_{r}\right)
$$

where $\bar{W}_{I_{0}}\left(k_{r}\right)$ is the volume-integrated wave packet at the peak intensity $I_{0}$

$$
\bar{W}_{I_{0}}\left(k_{r}\right)=\rho \int_{0}^{I_{0}} W_{I}\left(k_{r}\right)\left(\frac{\partial V}{\partial I}\right) d I
$$

with $W_{I}\left(k_{r}\right)$ being the wave packet for the laser pulse at a single intensity $I$.

\section{B. Wavelength dependence}

In a recent experiment, Colosimo et al. 48] reported electron spectra generated from Ar by infrared (IR) to mid-infrared (MIR) lasers with the same peak intensity, for wavelength of 800,1300, 2000 and $3600 \mathrm{~nm}$, respectively. The measured electron spectra with electron energies in units of $U_{p}$ are shown in Fig. 14, as well as the results from the QRS model above $4 U_{p}$ where volume integration effect has been included. First we note that there is a general agreement between the HATI spectra from the measurement and from the QRS model. For $800 \mathrm{~nm}$, the QRS underestimates the $4-5.5 U_{p}$ region which could be due to the resonantlike enhancement effect [49]. In the figure, the electron spectra from the different wavelengths are normalized near at threshold. One notes that the HATI yields decrease rapidly with increasing wavelength. Such decrease is also familiar in the HHG spectra [50], empirically estimated to decrease like $\lambda^{-5.5}$. For longer wavelength lasers, the excursion distance and the return time of the electron after tunnel ionization both scale with the wavelength, thus increase the effect of broadening in the returning wave packet.

From Fig. 13, we note that the slope of the electron spectra appears to flatten out considerably in going from $1300 \mathrm{~nm}$ to $2000 \mathrm{~nm}$. To understand the origin of the slope change, we show in Fig. 14(a) the returning electron wave packet obtained from the QRS model. The momentum of the wave packet is expressed in units of $A_{0}$ of the laser pulse at the focus center. We note the rapid oscillations are due to the ATI peaks. In Fig. 14(b) the volume integrated wave packets are shown. We note that they are mostly flat above $4 U_{p}$. According to the QRS model, Eq. (53), we thus expect that the slope change in the experimental data is due to the elastic scattering cross sections. In Fig. 15, we show the differential cross

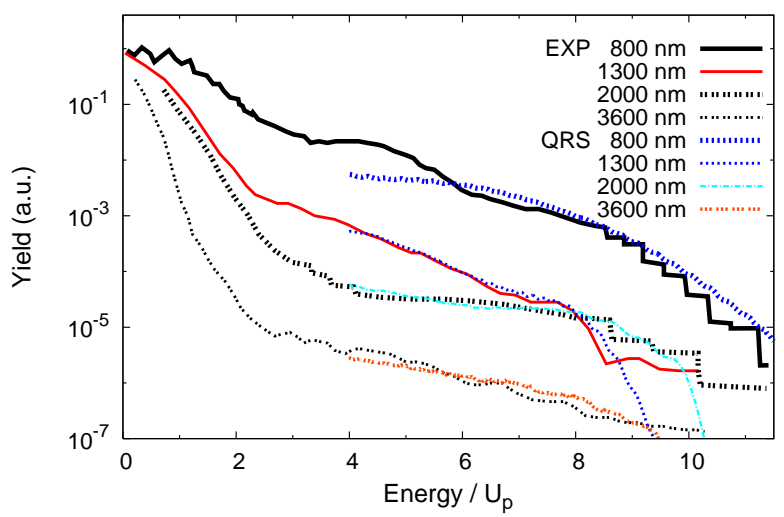

FIG. 13: (Color online) Comparison of experimental and QRS angle-integrated photoelectron energy spectra for single ionization of Ar by few-cycle laser pulses at the peak intensity of $0.8 \times 10^{14} \mathrm{~W} / \mathrm{cm}^{2}$ with wavelengths of $800 \mathrm{~nm}, 1300 \mathrm{~nm}$, $2000 \mathrm{~nm}$ and $3600 \mathrm{~nm}$, respectively. The experimental measurements are taken from [48]. The QRS results starting from $4 U_{p}$ are normalized to the experimental data individually at high energies to get best fit. Electron energies are expressed in units of $U_{p}$.
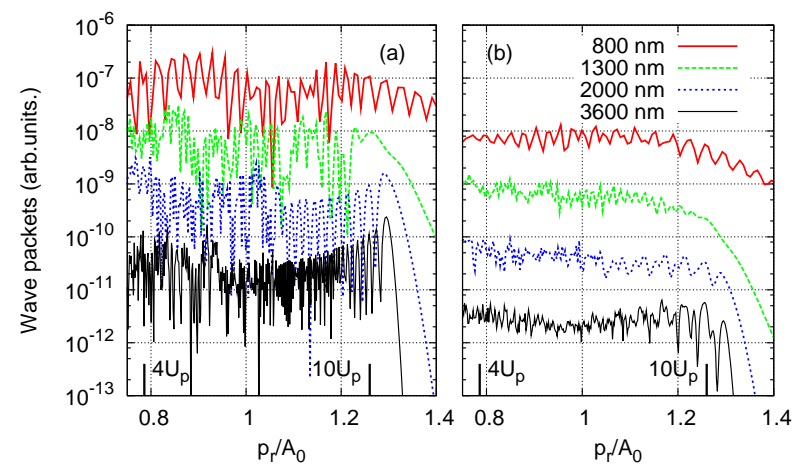

FIG. 14: (Color online) Wave packets at $800 \mathrm{~nm}, 1300 \mathrm{~nm}$, $2000 \mathrm{~nm}$ and $3600 \mathrm{~nm}$. (a) For single intensity of $0.8 \times 10^{14}$ $\mathrm{W} / \mathrm{cm}^{2}$; (b) Volume-integrated for the peak intensity of $0.8 \times$ $10^{14} \mathrm{~W} / \mathrm{cm}^{2}$ at the focal point of a Gaussian beam. See text.

sections of electron- $\mathrm{Ar}^{+}$collisions in the four momentum ranges for the returning electrons that contribute to the HATI spectra for the four wavelengths used. Note that the DCS peaks sharply at large angles near $180^{\circ}$ for the momentum range of 1.60-2.65 for the $2000 \mathrm{~nm}$ laser pulse. Such behavior of the DCS is responsible for the much flatter HATI spectra seen in Fig. 15 for the 2000 $\mathrm{nm}$ pulse. Note the similarity of the DCS in Fig. 15(c) in Ar, and the DCS in Xe in Fig. 12(a). The large DCS at angles close to $180^{\circ}$ is responsible for the much flatter energy dependence observed in the HATI spectra.

The above analysis shows that the QRS model not only can explain experimental results, but also offers a clear 

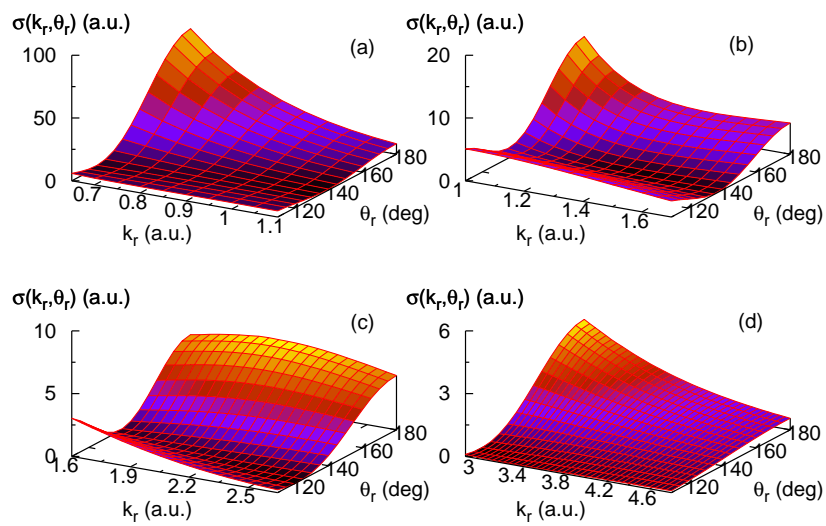

FIG. 15: (Color online) DCS's for elastic scattering of electron with $\mathrm{Ar}^{+}$at large scattering angles of $110^{\circ}-180^{\circ}$. The incident electron momenta are: (a) 0.65-1.10, (b) 1.00-1.75, (c) 1.60-2.65, and (d) 2.95-4.80, corresponding to the high energy plateau regions for $800 \mathrm{~nm}, 1300 \mathrm{~nm}, 2000 \mathrm{~nm}$ and $3600 \mathrm{~nm}$, respectively.

physical interpretation of the origin in the difference of the observed HATI spectra. We comment that in principle these HATI spectra can also be calculated by solving the TDSE. However, as shown in Colosimo et al. [48], such TDSE calculations for MIR lasers are very difficult due to their large long excursion distance and the need of a large box in the calculation for MIR laser pulses. Using the QRS, the calculation is much easier. In fact, the volume integrated returning wave packet is quite flat that one may even just approximate it by a constant. The QRS model then would predict that the slope seen in Fig. 13 is due to the integration of the DCS over the scattering angles. Note that by changing the peak intensity at the laser focus, the same flat plateau seen in Fig. 13 is expected to shift to different wavelength.

\section{Target and intensity dependence}

We also used the QRS model to simulate HATI spectra from some earlier experiments of Grasbon et al. 10]. Since in experiments the peak laser intensity is often determined approximately only, we perform the simulation by treating the peak laser intensity as a free parameter. We assume that all the electrons from the focal volume are collected in the experiment. We use the pulse duration and wavelength reported in the experiment. In Fig. 16(a) we compare the experimental HATI spectra measured with the ones obtained from the QRS model. Note that the peak intensity reported in the experiment is often different from the one that gives the best fit obtained from the QRS. In each spectra, we normalized the QRS result such that it gives best overall fit to the exper-
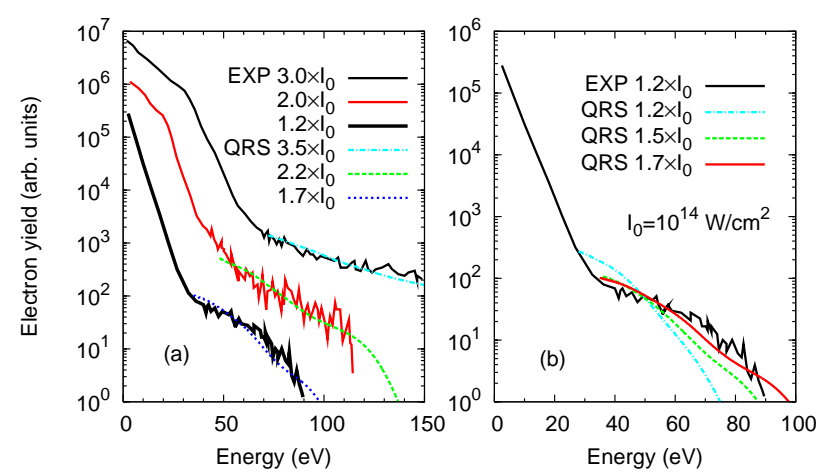

FIG. 16: (Color online) (a) Comparison of the total energy spectra of QRS with experimental measurements for Ar of [10] at different intensities; (b) Illustration of how to adjust intensity in the QRS simulation to fit experimental measurement. In the QRS calculations, volume effect has been included.
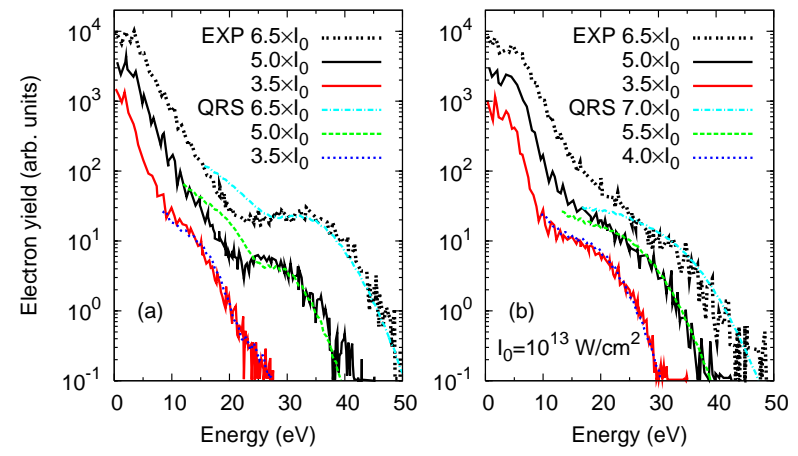

FIG. 17: (Color online) Comparison of the total electron energy spectra from QRS with the experimental measurements of [10]. (a) Xe; (b) Kr. In the QRS calculations, volume effect has been included.

imental data. Fig. 16(b) illustrates the example how the slope of the HATI spectra changes as the peak intensity at the focus is varied.

Similar analysis has been carried out for $\mathrm{Xe}$ and $\mathrm{Kr}$ targets, with the results shown in Fig. 17. In Kr, the agreement is very good for all three spectra. The small difference at the low energy end could be due to contributions from direct tunneling ionization. For Xe, we have been able to reproduce the outer part of the plateau well. At lower electron energies the experimental data show a deeper minimum for the two upper intensities which are not reproduced by the QRS simulation. The reason for this discrepancy is not clear at this time. In Fig. 18(a) we show the relative weights of the different peak intensities that contribute to the total ionization yields for the 

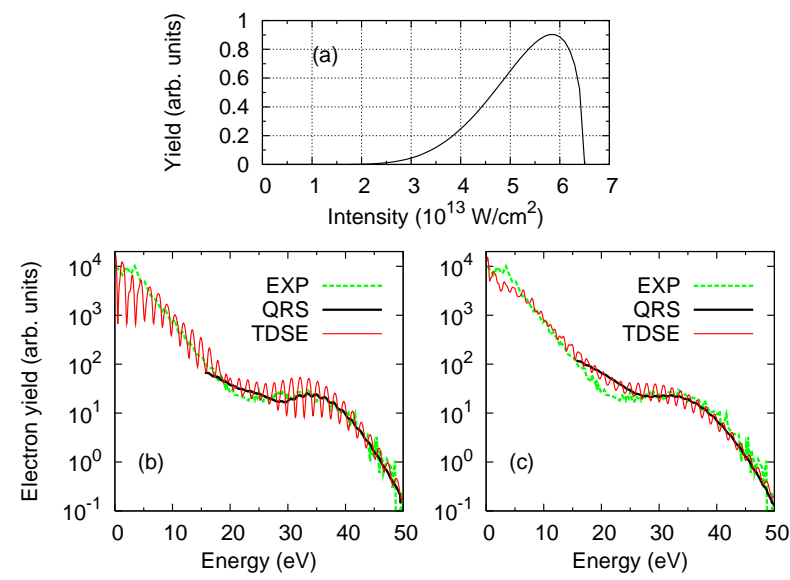

FIG. 18: (Color online) Single ionization of Xe by a 8 fs pulse at the peak intensity of $0.65 \times 10^{14} \mathrm{~W} / \mathrm{cm}^{2}$ with wavelength of $800 \mathrm{~nm}$. (a) Laser volume effect analysis showing the relative contributions of laser peak intensity to the generated electron spectra. (b) Comparison of QRS with TDSE at the single intensity of $0.59 \times 10^{14} \mathrm{~W} / \mathrm{cm}^{2}$; (c) Comparison of QRS with TDSE with volume effect included.

focal peak intensity of $0.65 \times 10^{14} \mathrm{~W} / \mathrm{cm}^{2}$. In Fig. 18(b), we show that the HATI spectra from the QRS agree well with the result from solving the TDSE at peak intensity of $0.59 \times 10^{14} \mathrm{~W} / \mathrm{cm}^{2}$, the intensity that contributes most to the electron yields, see Fig. 18(a).

Fig. 18(c) shows that volume integrated electron spectra calculated from TDSE and from the QRS agree well, but both cannot reproduce the deeper experimental minimum. This minimum occurs at about $20 \mathrm{eV}$, which is close to returning electron momentum of $k_{r}=0.67$, or electron energy of about $6 \mathrm{eV}$. At such a low energy, electron- $\mathrm{Xe}^{+}$elastic scattering cross sections may not be well described by the model potential approach. Recall that in QRS and TDSE, we treated Xe atom using a single active electron model. For electron-atom and electron-ion collisions, it is generally known that manyelectron correlation effect becomes more important as the collision energy decreases. (An example where the single electron approximation fails has been noted in the photodetachment of $H^{-}$by MIR lasers, see Zhou et al [43].) Thus one possible explanation for the failure of the QRS theory to reproduce the experimental observation in Fig. 17(a) is the need to include electron correlation effect in calculating $\mathrm{e}^{-}-\mathrm{Xe}^{+}$elastic scattering cross sections. While such calculations have been carried out using many-body perturbation theory [52, 53] in a number of cases, no such results have been reported for the present system. The fact that the minimum occurs at the same photoelectron energy in the experimental data at the two upper intensities, see Fig. 17(a), also offer a hint that the discrepancy is due to error in the DCS used. The minimum does not appear at the lowest intensity in Fig. 17(a) since the HATI yield drops rapidly in the same

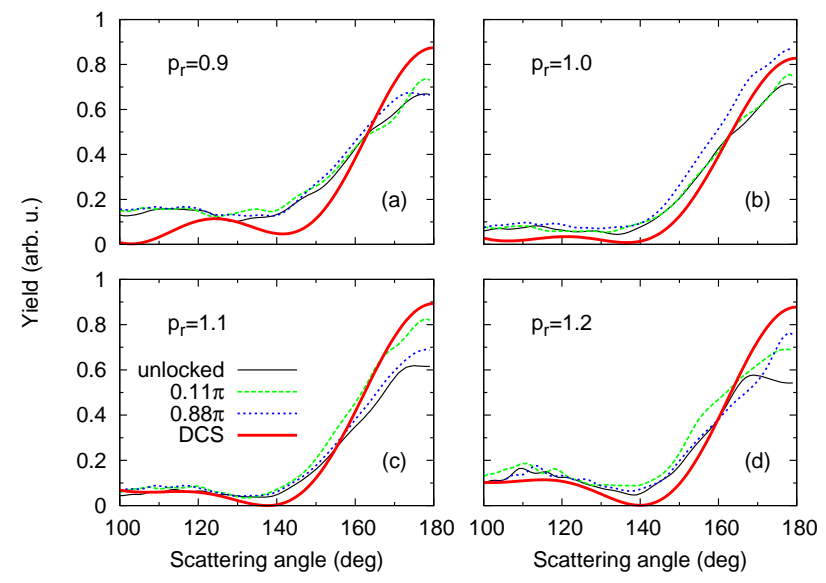

FIG. 19: (Color online) Comparison of the DCS's with electron yield extracted from the experimental measurements for Xe by laser pulses with different CEP's and unlocked CEP for (a) $k_{r}=0.9$, (b) $k_{r}=1.0$, (c) $k_{r}=1.1$, and (d) $k_{r}=1.2$. The CEP's are given in the figure for phase-locked pulses.

energy region.

\section{Extracting DCS from experimental electron spectra and Other Applications}

We have applied the QRS model to a number of other topics involving HATI electrons so far. In Chen et al. 25] the flatness of the HATI spectra vs electron energies observed for rare gas atoms and alkali atoms were investigated and interpreted in terms of the energy and angular dependence of the DCS, similar to the examples presented here. The QRS model has also been applied to retrieve the absolute value of the carrier-envelope-phase of few-cycle pulses, as well as the pulse duration and the peak laser intensity in Micheau et al. [28, 30]. Using the wave packet extracted from the HATI spectra and electron-impact ionization cross sections, the nonsequential double ionization of Ar has been obtained in Micheau et al. [31.

Following the earlier theoretical paper of Morishita et al. 24] in which DCS was extracted from the HATI spectra along the BRR, i.e., along $k_{r}=1.26 A_{0}$, the prediction was confirmed in two experiments [26, 27]. In these experiments, pulse durations of about $100 \mathrm{fs}$ and $8 \mathrm{fs}$ were used, respectively. As shown in Fig. 6(b), for such long pulses the extraction of DCS is difficult because of the interference in the wave packet. Interestingly, since the experimental electron spectra are collected from the whole focal volume, the oscillation in the volume-integrated wave packet at a given $k_{r}$ is smoothed out. Thus in effect, the success of retrieving the DCS in these two earlier experiments 26, 27] is based on the present QRS model, rather than the early theory of 24]. In other words, the effect of volume integration in the electron spectra ac- 
tually simplifies the retrieval of the DCS. Similarly, the DCS can also be extracted from ATI spectra generated by few cycle pulses. For these pulses, the returning electron wave packet depends on the carrier-envelope phase. One can obtain the DCS from experiments where the CEP is locked, or from measurements where the CEP is not locked. The extracted DCS should be the same based on the QRS model. In Fig. 19, we show the extracted DCS for Xe at four different electron momentum values, using the experimental data of Kling et al. [51]. The data extracted from these different data sets agree quite well, and they agree reasonably well with the theoretical DCS calculated from single active electron approximation. The theory shows slightly deeper minimum, but the angular resolution was not considered in the theoretical calculations. We emphasize that the extracted DCS should be independent of the lasers used.

\section{SUMMARY AND OUTLOOK}

In this paper we present a comprehensive QRS theory for describing the energy and momentum distributions of HATI electron spectra generated by intense laser pulses. Although HATI spectra have been interpreted in terms of rescattering concept since the 1990's, the QRS model is the first quantitative rescattering theory that can achieve accuracy comparable to those obtained from solving the TDSE. The essential ingredient of the QRS is governed by Eq. (1) which states that HATI electron momentum distributions can be expressed as the product of a returning electron wave packet $W\left(k_{r}\right)$ and the elastic differential cross sections $\sigma\left(k_{r}, \theta_{r}\right)$ between free electrons and the target ion. The validity of the QRS model is carefully tested against results obtained from solving TDSE using atomic targets in the single electron approximation. Here are a number of the most notable results:

(i) The wave packet $W\left(k_{r}\right)$ is mostly independent of the target, and can be calculated from the 2nd-order strong field approximation theory. All the laser dependence in the HATI spectra is governed by $W\left(k_{r}\right)$. The target structure enters through the DCS, $\sigma\left(k_{r}, \theta_{r}\right)$.

(ii) Using a practical QRS model, HATI spectra can be calculated with accuracy comparable to those obtained from solving TDSE, but with saving of computing time by a factor of thousands. This is particularly important when one wants to compare calculations with experimental measurements where repeated calculations have to be done for many intensities. With the QRS, as we demonstrated in Section IV, quantitative comparison of HATI spectra between theory and experiment is now possible. The QRS theory also is capable of checking consistency of different experiments.

(iii) The QRS model allows the extraction of the returning electron wave packet (in the laser field) based on HATI spectra at the end of the laser pulse, instead of us- ing classical simulations or theoretical models when the electron is still in the laser field. Our extracted $W\left(k_{r}\right)$ reflects the interference due to returning electrons with long- vs short-trajectories, as well as interference from electrons released from different optical cycles.

(iv) The QRS model allows one to separate the role of laser pulses which is contained in $W\left(k_{r}\right)$, and the role of the target which is contained in $\sigma\left(k_{r}, \theta_{r}\right)$. By calculating $W\left(k_{r}\right)$ using SFA2, the difficult part of the nonlinear laser-atom interaction is avoided. This accounts for the major saving in computer time in the QRS as compared to TDSE. The conceptual separation of the nonlinear effect of lasers on the wave packet and the structural information contained in the DCS has many ramifications. In particular, it should be possible to generalize the QRS model to include many-electron effects, just by replacing $\sigma\left(k_{r}, \theta_{r}\right)$ with the DCS calculated including electron correlation effect. But most importantly, the QRS model offers an opportunity to calculate accurate HATI spectra for molecules.

For molecular targets, there are many possible important applications. One can use a pump pulse to impulsively align molecules [54]. By taking the HATI spectra at the time when the molecules are preferentially aligned or anti-aligned with respect to the polarization of the pump beam, the dependence of the DCS on the alignment of molecules can be obtained. Such measurements are beginning to emerge [55]. However, current interpretation of such experimental data, for molecules that are aligned [55] or not aligned [56], rely on intuitive simple physical model 55], or on the extension of SFA2 to molecular targets [57, 58]. As shown in the present paper, SFA2 is not expected to adequately describe the backscattering of the returning electrons by the molecular ions. Using the QRS, more accurate description of the HATI spectra is possible if the DCS from electron-molecular ions collisions are available. Electronmolecule as well as electron-molecular ion collisions have been studied over the past few decades, both in experiments and in theory. A few general purpose codes are in existence [59, 60, 61, 62. . By adopting these codes to obtain the DCS needed for the QRS model, accurate HATI spectra from aligned molecules can be calculated.

Another potentially very important application is to use infrared lasers for dynamic imaging of a transient molecule [63]. Using a pump pulse to initiate a chemical reaction, the time evolution of the transient molecule, including the position of its constituent atoms, can be probed by measuring the HATI spectra by a probe laser. Today few-cycle pulses of duration of a few femtoseconds are readily available, and as indicated in Section IV, no CEP stabilization is needed in order to extract the DCS. From the dependence of the DCS with respect to the time delay, one may be able to extract the time evolution of the structure of the transient molecule, thus achieving dynamic chemical imaging of molecules with temporal 
resolution of a few femtoseconds. The feasibility of extracting the structure of the target from the DCS has already been demonstrated for rare gas atoms using a simple genetic algorithm 32]. Extension of the method to molecular targets is likely to be straightforward. Thus while the idea of using laser induced electron diffraction for imaging the structure of molecules had been proposed since 1996 [64], due to the relatively low energies of the returning electrons, the standard electron diffraction theory [65] is not applicable. On the other hand, with the QRS proposed here, and with the implementation of the state-of-the-art electron-molecule scattering codes, we believe that the fundamental theoretical tools needed for retrieving the structure of transient molecules based on laser-induced high-energy photoelectron spectra have been established.

Before closing, perhaps it is fitting to mention that an expression similar to Eq. (1) has been used to explain high-energy electron emission in the forward directions in energetic ion-atom collisions, such as the collision between $\mathrm{Cu}^{5+}$ with $\mathrm{H}_{2}$ in Liao et al. [66]. These electrons are best understood in the projectile frame where a beam of "free" electrons from $\mathrm{H}_{2}$ are incident on the $\mathrm{Cu}^{5+}$ ion. When these electrons are backscattered by the ion, they emerge as high-energy electrons in the laboratory frame. In this model, the beam of "free" electrons from $\mathrm{H}_{2}$ is treated as a wave packet, represented by the Compton profile of the target, in the projectile frame, similar to the returning wave packet $W\left(k_{r}\right)$ in the present QRS model. The transformation of momentum from the projectile frame to the laboratory frame in ion-atom collision is equivalent to the $-A_{r}$ term in the present paper, see Eqs. (48) and (49). In fact the similarity is so close that we quote the abstract of [66] here: "We present a method of deriving energy and angle-dependent electron-ion elastic scattering cross sections from doubly differential cross sections for electron emission in ion-atom collisions. By analyzing the laboratory frame binary encounter electron production cross sections in energetic ion-atom collisions, we derive projectile frame differential cross sections for electrons elastically scattered from highly charged projectile ions in the range between $60^{\circ}$ and $180^{\circ}$. The elastic scattering cross sections are observed to deviate strongly from the Rutherford cross sections for electron scattering from bare nuclei. They exhibit strong RamsauerTownsend electron diffraction in the angular distribution of elastically scattered electrons, providing evidence for the strong role of screening played in the collision." Indeed, electron scattering experiments can be carried out without directly preparing a well-collimated electron beam!

\section{ACKNOWLEDGMENT}

This work was supported in part by Chemical Sciences, Geosciences and Biosciences Division, Office of Basic Energy Sciences, Office of Science, US Department of Energy. T. M. is also supported by a Grant-In-Aid for Scientific Research (C) from the Ministry of Education, Culture, Sports, Science and Technology, Japan, and by the Japanese Society for the Promotion of Science (JSPS) Bilateral joint program between the U.S. and Japan.

[1] P. Agostini, F. Fabre, G. Mainfray, G. Petite, and N. K. Rahman, Phys. Rev. Lett. 42, 1127 (1979).

[2] R.R. Freeman, P. Bucksbaum, H. Milchberg, S. Darack, D. Scumacher, and M. E. Geusic, Phys. Rev. Lett. 59, 1092 (1987).

[3] R. Wiehle, B. Witzel, H. Helm, and E. Cormier, Phys. Rev. A 67, 063405 (2003).

[4] A. Rudenko, K. Zrost, C. D. Schröter, V. L. B. de Jesus, B. Feuerstein, R. Moshammer, and J. Ullrich, J. Phys. B 37, L407 (2004).

[5] C. M. Maharjan, A. S. Alnaser, I. Litvinyuk, P. Ranitovic, and C. L. Cocke, J. Phys. B 39, 1955 (2006).

[6] Zhangjin Chen, Toru Morishita, Anh-Thu Le, M. Wickenhauser, X. M. Tong, and C. D. Lin, Phys. Rev. A 74, 053405 (2006).

[7] D. C. Arbó, S. Yoshida, E. Persson, K. I. Dimitriou, and J. Burgdöorfer, Phys. Rev. Lett. 96, 143003 (2006).

[8] Toru Morishita, Zhangjin Chen, Shinichi Watanabe, and C. D. Lin, Phys. Rev. A 75, 023407 (2007).

[9] B. Yang, K. J. Schafer, B. Walker, K. C. Kulander, P. Agostini, and L. F. DiMauro, Phys. Rev. Lett. 71, 3770 (1993).

[10] F. Grasbon, G. G. Paulus, H. Walther, P. Villoresi, G. Sansone, S. Stagira, M. Nisoli, and S. D. Silvestri, Phys. Rev. Lett. 91, 173003 (2003).

[11] M. B. Gaarde, K. J. Schafer, K. C. Kulander, B. Sheehy, D. Kim, and L. F. DiMauro, Phys. Rev. Lett. 84, 2822 (2000)

[12] G. G. Paulus, W. Nicklich, Huale Xu, P. Lambropoulos, and H. Walther, Phys. Rev. Lett. 72, 2851 (1994).

[13] G. G. Paulus, W. Becker, W. Nicklich, and H. Walther, J. Phys. B 27, L703 (1994).

[14] D. B. Milošević, E. Hasović, M. Busuladžić, A. Gazibegović-Busuladžić, and W. Becker, Phys. Rev. A 76, 053410 (2007).

[15] H. G. Muller and F. C. Kooiman, Phys. Rev. Lett. 81, 1207 (1998).

[16] H. G. Muller, Phys. Rev. A 60, 1341 (1999); Phys. Rev. Lett. 83, 3158 (1999).

[17] J. Wassaf, V. Véniard, R. Taïeb, and A. Maquet, Phys. Rev. Lett. 90, 013003 (2003); Phys. Rev. A 67, 053405 (2003).

[18] R. M. Potvliege and S. Vučič, Phys. Rev. A 74, 023412 (2006).

[19] R. Kopold, W. Becker, M. Kleber, and G. G. Paulus, J. Phys. B 35, 217 (2002).

[20] Tongy B. Borca, M. V. Frolov, N. L. Manakov, and A. F. Starace, Phys. Rev. Lett. 88, 193001 (2002). 
[21] G. G. Paulus, F. Lindner, H. Walther, A. Baltuška, E. Goulielmakis, M. Lezius, and F. Krausz, Phys. Rev. Lett. 91, 253004 (2003).

[22] P. B. Corkum, Phys. Rev. Lett. 71, 1994 (1993).

[23] K. J. Schafer, Baorui Yang, L. F. DiMauro, and K. C. Kulander, Phys. Rev. Lett. 70, 1599 (1993).

[24] Toru Morishita, Anh-Thu Le, Zhangjin Chen, and C. D. Lin, Phys. Rev. Lett. 100, 013903 (2008).

[25] Zhangjin Chen, Anh-Thu Le, Toru Morishita, and C. D. Lin, J. Phys. B (submitted).

[26] M. Okunishi, T. Morishita, G. Prümper, K. Shimada, C. D. Lin, S. Watanabe, and K. Ueda, Phys. Rev. Lett. 100, 143001 (2008).

[27] D. Ray, B. Ulrich, I. Bocharove, C. Maharjan, P. Ranitovic, B. Gramkow, B. Gramkow, M. Magrakvelidze, S. De, I. V. Litvinyuk, A. T. Le, T. Morishita, C. D. Lin, G. G. Paulus, and C. L. Cocke, Phys. Rev. Lett. 100, 143002 (2008).

[28] S. Micheau, Zhangjin Chen, A.-T. Le, J. Rauschenberger, M. F. Kling, and C. D. Lin, Phys. Rev. Lett. (submitted).

[29] Zhangjin Chen, Toru Morishita, Anh-Thu Le, and C. D. Lin, Phys. Rev. A 76, 043402 (2007).

[30] Samuel Micheau, Zhangjin Chen, Toru Morishita, AnhThu Le, and C. D. Lin, J. Phys. B (submitted).

[31] Samuel Micheau, Zhangjin Chen, Toru Morishita, AnhThu Le, and C. D. Lin, Phys. Rev. A (accepted).

[32] Junliang Xu and C. D. Lin, Phys. Rev. A (submitted).

[33] Anh-Thu Le, Toru Morishita, and C. D. Lin, Phys. Rev. A 78, 023814 (2008).

[34] Anh-Thu Le, R. Della Picca, P. D. Fainstein, D. A. Telnov, M. Lein, and C. D. Lin, J. Phys. B 41, 081002 (2008).

[35] X. M. Tong and C. D. Lin, J. Phys. B 38, 2593 (2005).

[36] R. H. Garvey, C. H. Jackman, and A. E. S. Green, Phys. Rev. A 12, 1144 (1975).

[37] D. O. Harris, G. G. Engerholm, and W. D. Gwinn, J. Chem. Phys. 43, 1515 (1965).

[38] A. S. Dickinson and P. R. Certain, ibid. 49, 4209 (1968).

[39] J. C. Light and R. B. Walker, ibid. 65, 4272 (1976).

[40] X. M. Tong and Shih-I Chu, Chem. Phys. 217, 119 (1997).

[41] D. B. Milošević, G. G. Paulus, D. Bauer, and W. Becker, J. Phys. B 39, R203 (2006)

[42] E. Hasovic, M. Busuladžić, A. Gazibegović-Busuladžić, D. B. Milošević, and W. Becker, Laser Phys. 17, 376 (2007).

[43] XiaoXin Zhou, Zhangjin Chen, Toru Morishita, Anh-Thu Le, and C. D. Lin, Phys. Rev. A 77, 053410 (2008).

[44] L. I. Schiff, Quantum Mechanics, p. 145, 3rd Edition, McGraw-Hill, New York, (1968).

[45] C. J. Joachain, Quantum Collision Theory, p. 144, 3rd Edition, North-Holland, Amsterdam, (1983).

[46] S. J. Brotton, P. McKenna, G. Gribakin and I. D.
Williams, Phys. Rev. A 66, 062706 (2002).

[47] S. Augst, D. D. Meyerhofer, D. Strickland, and S. L. Chin, J. Opt. Soc. Am. B 8, 858 (1991).

[48] P. Colosimo, G. Doumy, C. I. Blaga, J. Wheeler, C. Hauri, F. Catoire, J. Tate, R. Chirla, A. M. March, G. G. Paulus, H. G. Muller, P. Agostini, and L. F. Dimauro, Nature Phys., 4, 386 (2008).

[49] Zhangjin Chen, M. Okunishi, K. Shimada, G. Prümper, T. Morishita, C. D. Lin, and K. Ueda, Phys. Rev. A (submitted).

[50] J. Tate, T. Auguste, H. G. Muller, P. Salières, P. Agostini, and L. F. DiMauro, Phys. Rev. Lett. 98, 013901 (2007).

[51] M. F. Kling, J. Rauschenberger, A. J. Verhoef, E. Hasović, T. Uphues, D. B. Milošević, H. G. Muller, and M. J. J. Vrakking, New J. Phys. 10, 025024 (2008).

[52] W. R. Johnson and C. Guet, Phys. Rev. A 49, 1041 (1994).

[53] B. A. Huber, C. Ristori, C. Guet, D. Kchler, and W. R. Johnson, Phys. Rev. Lett. 73, 2301 (1994).

[54] H. Stapelfeldt and T. Seidemann, Rev. Mod. Phys. 75, 543 (2003).

[55] M. Meckel, D. Comtois, D. Zeidler, A. Staudte, D. Pavičić, H. C. Bandulet, H. Pépin, J. C. Kieffer, R. Dörner, D. M. Villeneuve, and P. B. Corkum, Science 320, 1478 (2008).

[56] M. Okunishi, R. Itaya, K. Shimada, G. Prümper, K. Ueda, M. Busuladžić, A. Gazibegović-Busuladžić, D. B. Milošević, and W. Becker, J. Phys. B 41, 21004 (2008).

[57] M. Busuladžić, A. Gazibegović-Busuladžić, D. B. Milošević, and W. Becker, Phys. Rev. Lett. 100, 203003 (2008).

[58] M. Busuladžić, A. Gazibegović-Busuladžić, D. B. Milošević, and W. Becker, Phys. Rev. A 78, 033412 (2008).

[59] Robert R. Lucchese and Vincent McKoy, Phys. Rev. A 24, 770 (1981).

[60] R. E. Stratmann and Robert R. Lucchese, J. Chem. Phys. 102, 8493 (1995).

[61] S. Tonzani, Comput. Phys. Commun. 176, 146 (2007).

[62] S. Tonzani and C. H. Greene, J. Chem. Phys. 124, 054312 (2006).

[63] Toru Morishita, Anh-Thu Le, Zhangjin Chen, and C. D. Lin, New J. Phys. 10, 025011 (2008).

[64] T. Zuo, A. D. Bandrauk, and P. B. Corkum, Chem. Phys. Lett. 259, 313 (1996).

[65] I. Hargittai and M. Hargittai, Stereochemical Applications of Gas-Phase Electron Diffraction (New York: VCH) (1988).

[66] C. Liao, S. Hagmann, C. P. Bhalla, S. R. Grabbe, C. L. Cocke, and P. Richard, Phys. Rev. A 59, 2773 (1999). 